\title{
ALMOST EQUICONTINUOUS TRANSFORMATION GROUPS
}

BY

\section{PING-FUN LAM}

ABSTRACT. A class of transformation groups called strictly almost equicontinuous transformation groups is studied. Manifolds which carry such transformation groups are determined. Applications to related classes are obtained.

0 . Introduction. We consider an arbitrary group of homeomorphisms $G$ of a metric space $X$. Let $N(X, G)$ be the set of points in $X$ where the family of maps $G$ fail to be equicontinuous. The set $N(X, G)$ is an object of study in this paper. If we provide $G$ with a suitable topology, then $G$ becomes a topological group $T$ and the group of mappings $G$ induces, in a canonical way, a transformation group $(X, T)$. The set $N(X, G)$ is now renamed $N(X, T)$. It has been generally known in literature that the set $N(X, T)$ possesses certain interesting topological properties and it plays a role in determining isomorphic classes of actions of $T$ on $X$. A more wellknown result is that of Homma-Kinoshita [11], which shows that if $X$ is locally compact, if $T$ is the additive group of integers and if the set $N(X, T)$ is finite and does not disconnect $X$, then $N(X, T)$ has at most two points and the points are fixed points of $T$. The same conclusion is obtained by Kaul [15] and Gray-Roberson [9], which allow the finite set $N(X, T)$ to be a compact zero-dimensional set at the cost that $X$ is restricted to be locally connected. The theorems of Kaul [15] and Gray-Roberson [9] are consequences of Theorem 1.7 of this paper. See the proof of (1.17). Theorem 1.7, however, does not require that $X$ be locally connected. Gray-Roberson [9] also gives results for general acting groups $T$. Under the above hypothesis it is shown in [9] that there are at most two minimal sets in $N(X, T)$ and if $T$ is either connected or abelian, then $N(X, T)$ consists of one or two fixed points. Parting from this direction there are results on the topological conjugacy problem in terms of the set $N(X, T)$ (see Husch [13] and its references). For other recent developments see remarks at the end of the paper.

We consider the following two classes of transformation groups. Class I,

Received by the editors Norember 11, 1971 and, in revised form, June 15, 1972 and July 9, 1973.

AMS (MOS) subject classifications (1970). Primary 54H20.

Key words and phrases. Equicontinuity of maps, almost periodic point, one-point compactification, upper semicontinuous function, semiclosed set, homomorphi sm of transformation group, locally flat embedding, generalized Schoenflies theorem. 
$N(X, T)=\varnothing$. Class II, $\varnothing \neq N(X, T) \neq X$ and that $N(X, T)$ is sufficiently small in $X$ (for example if $N(X, T)$ is a 1-dimensional and $X$ is a 3-dimensional space). Class $I$ is called equicontinuous transformation groups. Generally speaking, Class II can be called strictly almost equicontinuous transformation groups in the large. We will refer to both I and II as almost equicontinuous transformation groups. In this paper we study Class II. We investigate the set $N(X, T)$ and some related problems such as to determine manifolds which carry such transformation groups. When $N(X, T)$ is zero-dimensional, a rigorous definition for Class II will be given in $\$ 1$ (cf. Definition 1.6). The definition is more general than that given by Gray-Roberson [9]. We mention that Class I and Class II have opposite dynamical properties in many respects. For the analysis of such dynamical properties, we refer the interested readers to a forthcoming paper by us on dynamical properties of almost equicontinuity.

One of the main results that we obtain is Theorem 1.7, which roughly asserts that when the set $N(X, T)$ is zero-dimensional it must belong to one of five special types given in the theorem. No assumption of local connectedness of $X$

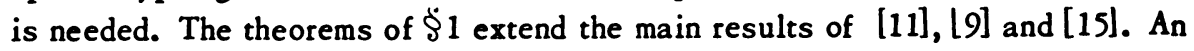
attempt is made in $\$ 2$ to pass these results to Class II in general. Only the more immediate properties which can be inferred from the method are stated in this paper. For more indirect applications see [14]. In $\$ 3$ we show that manifolds of dimension at least 2 which carry zero-dimensional $N(X, T)$ such that $\mathrm{Cl}(x T)$ is compact for each $x \in N(X, T)$ are necessarily of four standard topological types. Results on manifolds when $N(X, T)$ is of Class II but not of zero-dimension are now available in recent literature (cf. for example [5]).

This paper also serves to prove and extend some theorems which we have announced in [19]. They are Theorems 2, 4, 5, 6 and 9 of [19]. Theorem 2 of [19] is given as Theorems 1.7 and 1.22 here. Theorem 5 of $[19]$ is given in generalized form as Theorem 1.28. The generalization is that the original hypothesis is replaced by a weaker one, which becomes the same as that of Theorem 2 of [19]. Theorem 6 of [19] has been extended in many respects (cf. remarks at the beginning of $\$ 2$ ). It is proved in Theorem 2.12. Theorem 9 of [19] is proved in Theorem 3.4, with a correction on statement (IV). Theorem 4 of [19] follows from results of $\$ 3$.

The paper is written in a series with [20] preceding it and followed by a forthcoming work by us on dynamical properties of almost equicontinuity. Our work [20] provides the foundation for a property which we call indivisibility and, most important of all, obtains sufficient topological conditions for this abstract notion, which is to be used in definitions here.

Standing hypothesis. All phase spaces of transformation groups are assumed to be metric spaces unless otherwise stated. A chosen metric is given to each such metric space. 
Standing notations:

(1) $(X, T)$ : a transformation group with phase space $X$ and acting group $T$. The assumption on the space $X$ follows that of standing hypothesis.

(2) $E(X)=E(X, T)$ : the set of all equicontinuous points of $(X, T)$ with respect to a prechosen metric of the metric space $X$. The complement, the set of all nonequicontinuous points of $(X, T)$, is denoted by $N(X)$ or $N(X, T)$. In Proposition 1.1 the notations are used more generally for uniform spaces.

(3) $X^{*}=X \cup\{\infty\}$ : the one-point compactification of $X$.

(4) SAE: strictly almost equicontinuous transformation groups (see Definition 1.6).

1. Strictly almost equicontinuous transformation groups. The objective of this section is to give a definition of strictly almost equicontinuous transformation groups (Definition 1.6), to give a classification theorem for this class (Theorem 1.7) and to simplify the classification when the phase spaces are noncompact (Theorem 1.28).

Let $X$ be a uniform and $T_{2}$ space with a prescribed uniformity $\mathcal{U}$ and let $(X, T)$ be a transformation group. We say that a point $x \in X$ is equicontinuous (with respect to $T$ and $\mathcal{U}$ ) if for every $u \in \mathcal{U}$ there exists $v \in \mathcal{U}$ such that for $y \in X$

$$
(x, y) \in v \text { implies that }(x t, y t) \in u \text { for all } t \in T .
$$

We say that $x$ is nonequicontinuous otherwise. We let $E(X)$ or $E(X, T)$ denote the set of all equicontinuous points of $X$ under $T$. We let $N(X)$ or $N(X, T)$ denote the set of all nonequicontinuous points of $X$ under $T$.

Let $X$ be a metric space with a prescribed metric $d$ and let $(X, T)$ be a transformation group. We say that a point $x \in X$ is equicontinuous (with respect to $T$ and $d$ ) if for every $\epsilon>0$ there exists $\delta>0$ such that for $y \in X$

$$
d(x, y)<\delta \text { implies that } d(x t, y t)<\epsilon \text { for all } t \in T \text {. }
$$

The point $x$ is said to be nonequicontinuous otherwise.

When $X$ is a metric space with a prescribed metric $d$, the relation of the two previous definitions of equicontinuity is given as follows. Let $\mathcal{U}(d)$ be the uniformity of $X$ generated by the metric $d$, i.e. $U(d)$ has a base which consists of sets $V_{\epsilon}=\{(x, y) \epsilon X \mid d(x, y)<\epsilon\}$ for all $\epsilon>0$. Then $x \in X$ is equicontinuous with respect to $T$ and $d$ if and only if it is equicontinuous with respect to $T$ and $\mathcal{U}(d)$. These terminologies and properties are all well known.

In order to understand our formulation of the definition of strictly almost equicontinuous transformation groups, we first make a few preliminary remarks.

The condition of compact orbit closures for nonequicontinuous points. This condition is useful only for phase spaces $X$ which are noncompact. It ensures 
that each nonequicontinuous point of the metric is 'essential' in the sense that no nonequicontinuous point shall disappear when the prescribed metric of $X$ is replaced by another metric of $X$ which is compatible with the topology of $X$.

Let $(X, T)$ be a transformation group, where $X$ is a Tychonoff space. Let $S(X)$ be the set of all uniformities of $X$ which are compatible with the topology of $X$. For a chosen $\mathcal{U} \epsilon \mathcal{S}(X)$ it would be convenient here to denote $E(X)$ by $E(X \mid \mathcal{U})$ and $N(X)$ by $N(X \mid \mathcal{U})$.

Proposition 1.1 following concerns a few simple but important cases about how the set $E(X \mid \mathcal{U})$ is changed with respect to the change of $\mathcal{U}$.

1.1. Proposition. Let $(X, T)$ be a transformation group, where $X$ is Tychonoff. Let $S(X)$ be the uniformities of $X$ which are compatible with the topology of $X$. The following properties bold.

(1) Let $\mathcal{U}_{0} \in \mathcal{S}(X)$ be sucb that

$$
\mathrm{Cl}(p T) \text { is compact for every } p \in N\left(X \mid \mathrm{U}_{0}\right) \text {; }
$$

then $E(X \mid \mathcal{U}) \subset E\left(X \mid \mathcal{U}_{0}\right)$ for all $\mathrm{U} \in \mathcal{S}(X)$.

(2) Let the phase space $X$ be compact, or more generally let every orbit closure in $X$ be compact. Then $E\left(X \mid \mathcal{U}_{1}\right)=E\left(X \mid \mathcal{U}_{2}\right)$ for all $\mathcal{U}_{1}, \mathcal{U}_{2} \in \mathcal{S}(X)$.

(3) If $X$ is locally compact and $\mathrm{U}^{*}$ is the induced uniformity on $X$ from the (unique) uniformity of the one-point compactification $X^{*}$ of $X$, then $E(X \mid U) \subset$ $E\left(X \mid \mathcal{U}^{*}\right)$ for all $\mathcal{U} \epsilon S(X)$.

(4) If $X$ is a locally compact uniform space with a prescribed uniformity $U$ and if

$$
\mathrm{Cl}(p T) \text { is compact for every } p \in N(X \mid \mathcal{U})
$$

then the sets $E(X)$ and $N(X)$ are unchanged when $U$ is replaced by the induced uniformity from the one-point compactification of $X$.

(5) If $X$ is a locally compact separable metric space with a prescribed metric $d$ and if

$$
\mathrm{Cl}(p T) \text { is compact for every } p \in N(X) \text { with respect to } d \text {, }
$$

then the sets $E(X)$ and $N(X)$ are unchanged when $d$ is replaced by an induced metric from the one-point compactification of $X$ :

Proof. (1) Let $p \in N\left(X \mid \mathcal{U}_{0}\right)$. There exist a net $\left\{t_{i}\right\}$ in $T$, a net $\left\{x_{i}\right\}$ in $X$ and some $u \in \mathcal{U}_{0}$ such that

$$
\lim _{i} x_{i}=p, \quad\left(p t_{i}, x_{i} t_{i}\right) \notin u \text { for } t_{i} \in\left\{t_{i}\right\}
$$

By the compactness of $\mathrm{Cl}(p T)$ we may assume the existence of the limit $q=$ $\lim _{i} p t_{i}$. Consider $\mathcal{U} \in S(X)$. Choose a symmetric $v \in \mathcal{U}$ such that $q v^{3} \subset q u$. Then 
for $i$ sufficiently large we have $\left(p t_{i}, x_{i} t_{i}\right) \notin v$. For otherwise there exists arbitrary large $i$ such that $p t_{i} \in q v$ and then $x_{i} t_{i} \in q v^{2}$. Then $\left(p t_{i}, x_{i} t_{i}\right) \in v^{3} \subset u$ for such $i$. This contradicts (a). Hence $N\left(X \mid \mathcal{U}_{0}\right) \subset N(X \mid \mathcal{U})$. Hence $E(X \mid \mathcal{U}) \subset E\left(X \mid \mathcal{U}_{0}\right)$.

The proof of $(1)$ is completed.

(2) follows from (1). (3) is given in Proposition 2.11 of Lam [20]. Statements (4) and (5) are direct consequences of (1) and (3).

The proof of Proposition 1.1 is completed.

As a result of Proposition 1.1(4), (5) we have that if $X$ is locally compact and if points in $N(X)$ are so secured that they have compact orbit closures, then in analyzing the set $N(X)$ we may always assume that an induced metric or uniformity from the one-point compactification is being used. This adaptation will be useful for passing results from compact phase spaces to noncompact phase spaces (see (1.28) and (2.17)). As for proving results such as Theorem 1.7 we, however, find no advantage to select this special metric for $X$.

We consider a transformation group $(X, T)$.

Zero-dimensionality vis. total disconnectedness for the set $N(X)$. Let $X$ be a locally compact metric space. The set $N(X)$ is in general not a closed subset of $X$. Hence if the set $N(X)$ is totally disconnected, it is not a priori a zero-dimensional set. In the following we show that these two properties are equivalent. This equivalence permits us to use the more general hypothesis that $N(X)$ is totally disconnected in Definition 1.6, in place of the hypothesis that $N(X)$ is zero-dimensional. The total disconnectedness hypothesis is preferable for the technique of $\$ 2$. The proof of the equivalence is based on the following theorem of Lam [19].

1.2. Theorem. If $X$ is a metric space, then $N(X)$ is a countable union of closed invariant sets.

The proof of Theorem 1.2 is given in [20, (1.4)].

1.3. Proposition. Let $X$ be a locally compact metric space. Then $N(X)$ is zero-dimensional if and only if it is totally disconnected.

Proof. The necessity is clear. To prove the sufficiency we observe that every closed subset of $X$ is locally compact and every locally compact totally disconnected set is zero-dimensional. Hence if $N(X)$ is totally disconnected, then Theorem 1.2 implies that $N(X)$ is a countable sum of zero-dimensional closed sets. Such a set is known to be zero-dimensional (cf. Hurewicz-Wallman [12, p. 18]).

The proof of Proposition 1.3 is completed.

The condition that a set is indivisible by $T$. Condition (c) of Definition 1.6 is a nonstandard property. It requires that the set $E(X)$ be indivisible by $T$. We recall this special definition of indivisibility from [19]. Again a transformation group $(X, T)$ is under consideration. 
1.4. Definition. Suppose that $N(X)$ is zero-dimensional. A subset $Y$ of $X$ is said to be indivisible by $T$, if whenever there exist a sequence $\left\{t_{n}\right\}$ in $T$ and a point $y_{0} \in Y$ such that $\lim _{n \rightarrow \infty} y_{0} t_{n}=p \in N(X)$, then the sequences $\left\{y t_{n}\right\}$ have $p$ as a limit point for all $y \in Y$.

By considering suitable subsequences we see that $Y$ is indivisible by $T$ if and only if for every sequence $\left\{t_{n}\right\}$ in $T$ whenever there exists $y_{0} \in Y$ such that $\lim _{n \rightarrow \infty} y_{0} t_{n}=p \in N(X)$, then $\lim _{n \rightarrow \infty} y t_{n}=p$ for every $y \in Y$.

Although Definition 1.4 appears to be simple and abstract, it is shown in [19], [20] that there exist various topological conditions which give this property, and many other topological conditions, though very similar to the first set, do not give this property. The conditions of $[20]$ are summed up in Theorem 4.22 of [20], when $N(X)$ is zero-dimensional. In the form of metric phase spaces, as so assumed here, we have the following result from [20, Theorem 4.22].

1.5. Theorem. Suppose that $X$ is a locally compact metric space and that $N(X)$ is zero-dimensional. A sufficient condition for the set $E(X)$ to be indivisible by $T$ is that there is a dense subset $D$ of $E(X)$ so that one of the following cases occurs.

(1) $D$ is a semicontinuum.

(2) $D$ is locally compact, connected and invariant (the condition "invariant" can be omitted if $T$ is connected.

(3) $D$ is connected and either $D$ or $X$ is locally connected.

(4) $D$ is connected and $N(X)$ is either scattered or locally compact.

(5) $D$ is the union of a family $\left\{M_{\lambda}\right\}$ of connected sets, which are totally ordered by inclusions and which are disjoint from $\mathrm{Cl}(N(X))$.

The sufficient conditions are also sufficient conditions for a condition called " $E(X)$ is CC-indivisible by $T$ " in [19], which is slightly stronger than the present one when $X$ is not compact.

In application of Theorem 1.5 the set $D$ is often taken as $E(X)$ itself. There are, however, applications where it is known only that a dense subset $D$ of $E(X)$ rather than $E(X)$ itself has one of the five properties of Theorem 1.5 (see for example [14]). Each of the five properties in (1.5) implies that the set $E(X)$ is connected. However, an example is given in [20] which shows that there is a compact connected metric space $X$, a zero-dimensional $N(X, \mathbf{R})$, where $\mathbf{R}$ is the group of real numbers, a connected set $E(X, \mathbf{R})$, but that $E(X, \mathbf{R})$ is not indivisible by $T$. Note that for such counterexamples the set $N(X)$ is not closed, otherwise $E(X)$ would be locally compact and (2) of (1.5) would be satisfied with $D=E(X)$. Also for such counterexamples the space $X$ should not be locally connected according to (3) of (1.5). For general cases we do not have a single topological 
condition which is a suitable replacement for our indivisible condition of Definition 1.6 .

In [20] we define indivisibility in great generality which contains the present definition as a very special case. To be self-contained we will avoid using general theorems of [20] as much as possible. The essential use of [20] is the support of the property of indivisibility in Theorems 1.7, 2.12, 2.16 and 2.17 by topological sufficient conditions and for proofs of (1.27), (2.11), (2.17).

We are now in position to define our definition of Class II transformation groups (cf. Introduction) for the case that $N(X)$ is zero-dimensional.

1.6. Definition. A transformation group $(X, T)$ is said to be strictly almost equicontinuous (SAE) if $X$ is a locally compact connected metric space and the following conditions are satisfied:

(a) $N(X)$ is nonempty and totally disconnected.

(b) For each $p \in N(X)$ the set $\mathrm{Cl}(p T)$ is compact.

(c) The set $E(X)$ is indivisible by $T$.

Note that condition (b) is automatically satisfied if $X$ is compact.

The most important theorem of this section is the following one.

1.7. Theorem. If $(X, T)$ is strictly almost equicontinuous, then the set $N(X)$ is necessarily compact and zero-dimensional. Explicitly the set $N(X)$ must be of one of the following five kinds:

(i) a union of two fixed points $((X, T)$ is called type 2 for this case),

(ii) a fixed point (type 1a),

(iii) a minimal set which is homeomorphic to the Cantor set (type 1b),

(iv) a topological Cantor set which has a fixed point and all other orbits are dense in $N(X)$ (type 1c),

(v) an orbit of two elements (type 1d).

Any one of the last four types in (1.7) is called type 1. Our types are named as those of Kinoshita (cf. references in [17]), in which he considered only discrete flows, hence only type 2 and type la can occur (cf. (1.17)).

Theorem 1.7 is intended as an extension of part of the following classical theorem of Kerékjártó.

1.8. Theorem (Kerékjártó [16]). Let $f$ be an orientation preserving selfbomeomorphism of the 2-spisare $S^{2}$ whose powers (both positive and negative) are equicontinuous except for a finite number of points. Then

(A) The number of exceptional points is at most 2.

(B) For the nonexceptional point case $f$ is topologically conjugated to a rotation about a diameter; for the one exceptional point case $f$ is topologically conjugated to the homeomorphism obtained from extending the translation on the 
plane by adding the fixed point $\{\infty\}$; for the two exceptional points case $f$ is topologically conjugated to the homeomorphism obtained from extending the dilation $(f(x)=x / 2)$ on the plane by adding the fixed point $\{\infty\}$ (the proof of Kerékjártó has an error for the case when $f$ is periodic, cf. Eilenberg [6]).

Works related to (A) of Theorem 1.8 are [11], [9], [15] (cf. Introduction).

As for (B) of Theorem 1.8 it is also shown in [11] that if $X$ is compact and if $N(X)$ is a union of two fixed points $p$ and $q$, then

$$
\lim _{n \rightarrow \infty} f^{n}(x)=p \text { for all } x \neq q, \quad \lim _{n \rightarrow-\infty} f^{n}(x)=q \text { for all } x \neq p
$$

If $X$ is compact and $N(X)$ is a fixed point $z$, then

$$
\lim _{|n| \rightarrow \infty} f^{n}(x)=z \text { for all } x \in X .
$$

We also have a general result in this respect (cf. Theorem 1.22). This generalization is useful for applications in $\$ \$ 2,3$. For a strong generalization of (B) of (1.8) see [13] and its references. A discussion for the difficult case when $N(X)=\varnothing$ can be found in Montgomery-Zippin [21].

We begin to prepare some lemmas for the proof of Theorem 1.7. The first thing to do is to convert the equicontinuity property of $T$ to the equicontinuity property of sequences in $T$. By abuse of language we say that a sequence $\left\{t_{n}\right\}$ in $T$ is equicontinuous at $x \in X$ if the range of the sequence is equicontinuous at $x$.

In the following we assume that a transformation group $(X, T)$ is given. Let $d$ be the metric of $X$.

Let $\left\{t_{n}\right\}$ be a sequence in $T$ and $x \in X$. Then $\left\{t_{n}\right\}$ fails to be equicontinuous at $x$ if and only if there exists a subsequence $\left\{t_{n_{k}}\right\}$ of $\left\{t_{n}\right\}$, corresponding sequences $\left\{a_{n_{k}}\right\}$ and $\left\{b_{n_{k}}\right\}$ in $X$ and a number $r>0$ such that

$$
\lim _{k \rightarrow \infty} a_{n_{k}}=x=\lim _{k \rightarrow \infty} b_{n_{k}}, \quad d\left(a_{n_{k}} t_{n_{k}}, b_{n_{k}} t_{n_{k}}\right)>r
$$

As we shall see it is often convenient to replace a given sequence $\left\{t_{n}\right\}$ by its subsequence which satisfies (a). Such a subsequence is characterized as follows.

1.9. Remark. Let $\left\{t_{n}\right\}$ be a sequence in $T$ and $p \in X$. The following state. ments are equivalent:

(1) Every subsequence of $\left\{t_{n}\right\}$ fails to be equicontinuous at $p$.

(2) There exist two sequences $\left\{a_{n}\right\},\left\{b_{n}\right\}$ in $X$ and a number $r>0$ sucb that

$$
\lim _{n \rightarrow \infty} a_{n}=p=\lim _{n \rightarrow \infty} b_{n} ; \quad d\left(a_{n} t_{n}, b_{n} t_{n}\right)>r \text {. }
$$

It follows that if a sequence $\left\{t_{n}\right\}$ in $T$ fails to be equicontinuous at $p \in X$, 
then there exists a subsequence $\left\{t_{n_{k}}\right\}$ of $\left\{t_{n}\right\}$ so that every subsequence of $\left\{t_{n_{k}}\right\}$ fails to be equicontinuous at $p$. These properties for sequences of maps are well known.

1.10. Remark. Let $x \in X$. Then the following statements are equivalent:

(1) $x \in N(X)$.

(2) Some sequence in $T$ fails to be equicontinuous at $x$.

(3) $f_{\alpha}(x) \neq 0$ for some sequence $\alpha=\left\{t_{n}\right\}_{n=1}^{\infty}$ in $T$.

The function $f_{\alpha}$ of Remark $1.10(3)$ is an upper semicontinuous function $f_{a}$ : $X \rightarrow[0, \infty]$ defined by

$$
\left.f_{a}(x)=\inf \left\{\sup _{\operatorname{dia}}\left(S_{\epsilon}(x)_{t_{n}}\right) \mid n \geq 1\right\} \mid \epsilon>0\right\} \quad \text { (dia } \equiv \text { diameter), }
$$

where $S_{\epsilon}(x)$ is the $\epsilon$-open ball of $x$. The function has the property that $f_{a}(x)=0$ if and only if $a$ is equicontinuous at $x$. The function $f_{a}$ shows the analytic nature of the nonequicontinuous points of the sequence $a=\left\{t_{n}\right\}$. We will, however, give no further details of this function, other than remarking that some of the following lemmas can be phrased in terms of these functions. A crucial lemma follows.

1.11. Lemma. Let $X$ be locally compact and connected and let $N(X)$ be zerodimensional. If a sequence $\left\{t_{n}\right\}$ in $T$ fails to be equicontinuous at $p \in X$, whicb bas a compact orbit closure, then for some $a \in E(X)$ and for some subsequence $\left\{t_{n_{k}}\right\}$ of $\left\{t_{n}\right\}$ we bave $\lim _{k \rightarrow \infty} a t_{n_{k}}^{-1}=p$.

Proof. Clearly $p \in N(X)$. We first replace $\left\{t_{n}\right\}$ by a subsequence so that every subsequence of the subsequence is nonequicontinuous at $p$. The sequence $\left\{t_{n}\right\}$ is then characterized by Remark 1.9(2). By the compactness hypothesis of $\mathrm{Cl}(p T)$ we may further assume the existence of $q=\lim _{n \rightarrow \infty} p t_{n}$. By Theorem 1.2 we have that if $x \in N(X)$, then $\mathrm{Cl}(x T) \subset N(X)$. Hence $\mathrm{Cl}(p T) \subset N(X)$ and so $q \in N(X)$.

Now by the hypothesis that $N(X)$ is zero-dimensional at $p, p$ has arbitrarily small relatively open and closed neighborhoods $W$ in $N(X)$. Applying the hereditary normal property of the metric space $X$ to the sets $W$ and $N(X)-W$, both relatively closed in $N(X)$, we obtain arbitrarily small open neighborhoods $V$ of $p$ such that $\partial V \cap N(X)=\varnothing$. Since $X$ is locally compact, we may assume that $\mathrm{Cl}(V)$ is compact. Since $X$ is connected we may further assume that $\varnothing \neq \partial V \cap E(X)=\partial V$. We can then choose a sequence $\left\{V_{n}\right\}$ of open neighborhoods of $p$ which has the following properties:

$$
\begin{aligned}
& \mathrm{Cl}\left(V_{n}\right) \text { is compact, } \varnothing \neq \partial V_{n} \subset E(X) \text { for all } n, \\
& V_{1} \supset V_{2} \supset \cdots, \lim _{n \rightarrow \infty} \operatorname{dia}\left(V_{n}\right)=0, V_{1} \neq X .
\end{aligned}
$$

Since we have assumed that every subsequence of $\left\{t_{n}\right\}$ is nonequicontinuous at $p$, 
by Remark 1.9 there exists some $\epsilon>0$ such that

$$
\operatorname{dia}\left(V_{n} t_{n}\right)>\epsilon \text { for all } n=1,2, \cdots
$$

For the same reason that neighborhood $V$ of $p$ can be obtained by hypothesis we can choose open neighborhoods $U$ and $U_{1}$ of $q$ which have the following properties:

$$
\begin{aligned}
& \mathrm{Cl}(U) \text { is compact, } \operatorname{dia}(U)<\epsilon / 2, \mathrm{Cl}\left(U_{1}\right) \subset U, \\
& \varnothing \neq \partial U \subset E(X), \varnothing \neq \partial U_{1} \subset E(X) .
\end{aligned}
$$

The lemma will be proved by contradiction. We will first establish two assertions which are based on the assumption that the lemma is false. The contradiction and the proof of Lemma 1.11 are immediate consequences of the se assertions.

We denote the component of $V_{n}$ which contains $p$ by $A_{n}$. Then $A_{1} \supset A_{2} \supset$ $A_{3} \supset \cdots$

Assertion A. If Lemma 1.11 is false, then there exists an integer $r$ such that $A_{r} t_{n} \subset U_{1}$ for all $n>r$.

Proof of Assertion A. Assume that there exists no integer $r$ such that $A_{r} t_{n} \subset V_{1}$ for all $n \geq r$. Then for any positive integer $i$ there are integers $m_{i}, n_{i}$ such that $i \leq m_{i} \leq n_{i}$ and $A_{m_{i}}{ }_{n_{i}} \not \subset U_{1}$. Note that the sets $A_{m_{i}} t_{n_{i}}$ are connected. Since $p \in A_{m_{i}}$ for all $i$ and $p t_{n_{i}} \in U_{1}$ for sufficiently large $i$, we can use (c) to conclude that for all sufficiently large $i$ there exists $y_{i} \in A_{m_{i}}$ with $y_{i} t_{n_{i}} \in \partial U_{1}$. By the compactness of $\partial U_{1}$ we may assume the existence of the limit $\lim _{i \rightarrow \infty} y_{i} t_{n_{i}}=a \in \partial U_{1}$. Since $a \in E(X)$, we have

$$
\lim _{i \rightarrow \infty} a t_{n_{i}}^{-1}=\lim _{i \rightarrow \infty}\left(y_{i} t_{n_{i}}\right) t_{n_{i}}^{-1}=\lim _{i \rightarrow \infty} y_{i}=p \text {. }
$$

In this case Lemma 1.11 is true. Hence Assertion $A$ is valid.

Now by applying (a), (b) and (c) above we can obtain a sequence $\left\{x_{n} \mid n=\right.$ $1,2, \cdots\}$ with $x_{n} \in V_{n}$ and $x_{n} t_{n} \notin U$. For integers $k, n$ such that $1 \leq k \leq n$ we let $C_{k, n}$ be the component of $x_{n}$ in $\mathrm{Cl}\left(V_{k}\right)$. From a result of $K$. Menger [18, vol. 2, p. 172] we have that $C_{k, n} \cap \partial V_{k} \neq \varnothing$. Here the property $V_{1} \neq X$ in (a) is used.

Assertion B. If Lemma 1.11 is false and if $r$ is an integer given by Assertion $A$, there exists an integer $s \geq r$ such that $C_{s, n}{ }_{n} \cap U=\varnothing$ for all $n \geq s$.

The proof of Assertion B is similar to that of Assertion A, and we omit it. The relation between $U$ and $U_{1}$ in (c) is used in the proof.

We now assume that Lemma 1.11 is false. Let $s$ be an integer of Assertion B. The set $\lim \inf \left\{C_{s, n} \mid n=s, s+1, \ldots\right\}$ contains at least one point, namely $p$. Hence $C=\lim \sup \left\{C_{s, n} \mid n=s, s+1, \ldots\right\}$ is a connected set (cf. [18, vol. 2, p. 171]). Then $C \subset A_{s}$. We choose $z_{n} \in C_{s, n} \cap \partial V_{s}$ and let $z$ be a limit point of the sequence $\left\{z_{n} \mid n=s, s+1, \ldots\right\}$. Then $z \in \partial V_{s} \cap A_{s}$. In particular $z \in E(X)$. 
By Assertion A we have $z t_{n} \in U_{1}$ for all $n \geq r$. Hence for $z_{n}$ sufficiently near $z$ we have $z_{n} t_{n} \in U_{1}$. This would contradict Assertion $B$.

The proof of Lemma 1.11 is completed.

1.12. Lemma. Let $(X, T)$ be SAE (see Definition 1.6). If a sequence $\left\{t_{n}\right\}$ in $T$ is sucb that for a point $p_{1} \in X$, every subsequence is nonequicontinuous at $p_{1}$, then $\left\{t_{n}\right\}$ is equicontinuous at all points of $X$ other than $p_{1}$.

Proof. Let $p$ be an arbitrary point in $X$ at which $\left\{t_{n}\right\}$ is nonequicontinuous. We show $p=p_{1}$. By Proposition 1.3 the hypothesis of Lemma 1.11 holds. By (1.11) there exists a subsequence $\left\{t_{n_{k}}\right\}$ of $\left\{t_{n}\right\}$ and some $a \in E(X)$ such that $\lim _{k \rightarrow \infty} a t_{n_{k}}^{-1}=p$. By hypothesis the sequence $\left\{t_{n_{k}}\right\}$ is nonequicontinuous at $p_{1}$. If we apply (1.11) with $p$ replaced by $p_{1}$ and with $\left\{t_{n}\right\}_{\text {replaced by }}\left\{t_{n_{k}}\right\}$ we find a subsequence $\left\{s_{i}\right\}$ of $\left\{t_{n_{k}}\right\}$ and some $b \in E(X)$ such that $\lim _{i \rightarrow \infty} b s_{i}^{-1}=p_{1}$. By the hypothesis that $E(X)$ is indivisible by $T$ (cf. Definition 1.4), applied on the sequence $\left\{s_{i}^{-1}\right\}$, we must also have that $\lim _{i \rightarrow \infty} a s_{i}^{-1}=p_{1}$. This together with $\lim _{k \rightarrow \infty} a t_{n_{k}}^{-1}=p$ above imply that $p_{1}=p$.

The proof of Lemma 1.12 is completed.

1.13. Theorem. Let $(X, T)$ be strictly almost equicontinuous. The only possible relatively closed invariant subsets of $N(X)$ are the set $N(X)$ itself, the empty set or a fixed point.

Proof. Let $M$ be relatively closed and invariant in $N(X)$ and suppose that $M \neq N(X)$ and $M \neq \varnothing$. Let $p \in N(X)-M$. There exists a sequence $\left\{t_{n}\right\}$ in $T$ which fails to be equicontinuous at $p$. According to Lemma 1.11 there exist some $a \in E(X)$ and a subsequence $\left\{s_{k}\right\}$ of $\left\{t_{n}\right\}$ such that $\lim _{k \rightarrow \infty} a s_{k}^{-1}=p$. Since $E(X)$ is assumed to be indivisible by $T$, we have that $\lim _{k \rightarrow \infty} x s_{k}^{-1^{k}}=p$ for all $x \in E(X)$. Now let $z$ be an arbitrary point in $M$. Since $M$ is relatively closed in $N(X)$, we have some $\epsilon>0$ such that $S_{\epsilon}(p) \cap \mathrm{Cl}(M)=\varnothing$, where $S_{\epsilon}(p)$ is the $\epsilon$-open ball of $p$. Since $X$ is connected and $N(X)$ is zero-dimensional, there exist points in $E(X)$ which are arbitrarily near $z$. Since $M$ is invariant, all cluster points of the sequence $\left\{z s_{k}\right\}$ are in $\mathrm{Cl}(M)$. It follows that every subsequence of $\left\{s_{k}\right\}$ is nonequicontinuous at $z$. According to Lemma 1.12 the set $M$ must be a single point. Since $M$ is invariant, the point is a fixed point.

The proof of Theorem 1.13 is completed.

1.14. Lemma. Let $(X, T)$ be SAE. If a sequence $\left\{t_{n}\right\}$ in $T$ is nonequicontinuous at $p \in X$, there exists a subsequence $a=\left\{s_{k}\right\}$ of $\left\{t_{n}\right\}$ such that $\lim _{k \rightarrow \infty} x s_{k}^{-1}=p$ for all $x \in X$, except possibly for a set $N_{\alpha^{-1}}$. The set $N_{a^{-1}}$ bas at most one element and can be characterized as the set of points where $\left\{s_{k}^{-i}\right\}$ is nonequicontinuous. 
Proof. According to Lemma 1.11 there exist a subsequence $\left\{t_{n_{k}}\right\}$ of $\left\{t_{n}\right\}$ and some $a \in E(X)$ such that $\lim _{k \rightarrow \infty} a t_{n_{k}}^{-1}=p$. Since the set $E(X)$ is assumed to be indivisible by $T$, we have $\lim _{k \rightarrow \infty} x t_{n_{k}}^{-1}=p$ for all $x \in E(X)$. Note that the set $E(X)$ is dense in $X$. Suppose that the sequence $\left\{t_{n_{k}}^{-1}\right\}$ is equicontinuous at every point of $X$; then we may let $\left\{s_{k}\right\}$ be $\left\{t_{n_{k}}^{-1}\right\}$ and then Lemma 1.14 holds. In this case $N_{a^{-1}}=\varnothing$ and $\lim _{k \rightarrow \infty} x s_{k}^{-1}=p$ for all $x \in X$. Assume now that there is a point $q \in X$ at which $\left\{t_{n_{k}}^{-1}\right\}$ is nonequicontinuous. We let $\left\{s_{k}^{-1}\right\}$ be a subsequence of $\left\{t_{n_{k}}^{-1}\right\}$ so that every subsequence of $\left\{s_{k}^{-1}\right\}$ is nonequicontinuous at q. According to Lemma 1.12 the sequence $\left\{s_{k}^{-1}\right\}$ is then equicontinuous everywhere except at the point $q$. Since $\lim _{k \rightarrow \infty} x s_{k}^{-1}=p$ for $x \in E(X)$ and $E(X)$ is dense in $X-\{q\}$, we have $\lim _{k \rightarrow \infty} x s_{k}^{-1}=p$ for $x \in X-\{q\}$.

The proof of Lemma 1.14 is completed.

We state a weak form of Lemma 1.14 for the convenience of application in the sequel.

1.15. Lemma. Let $(X, T)$ be SAE. If $p \in N(X)$, then there exists a sequence $\left\{u_{i}\right\}$ in $T$ such that $\lim _{i \rightarrow \infty} x u_{i}=p$ for all $x \in X$, except possibly for a point of $N(X)$.

1.16. Proof of Theorem 1.7. As a result of Theorem 1.2 we have that $\mathrm{Cl}(p T) \subset N(X)$ for all $p \in N(X)$. We choose an arbitrary $p \in N(X)$ and let $M=$ $\mathrm{Cl}(p T)$. If $M=N(X)$, then clearly $N(X)$ is compact and zero-dimensional. If $M \neq N(X)$, then $M$ is a fixed point $p$ according to Theorem 1.13. For the latter case we take an arbitrary $q \in N(X), q \neq p$, if any such point exists. Again if $\mathrm{Cl}(q T) \neq N(X)$, then $q$ is a fixed point; hence $N(X)=\{p\} \cup\{q\}$ by (1.13). We have thus shown that $N(X)$ is compact and zero-dimensional. In addition we have shown that if $N(X)$ has a fixed point $p$ then the set $A=N(X)-\{p\}$ is either empty, a fixed point or that it consists of points each of whose orbits is dense in $N(X)$. If $N(X)$ has no fixed points, then every orbit in $N(X)$ is dense in $N(X)$.

We now claim that if the space $N(X)$ has an isolated point, then the number of points in $N(X)$ is at most 2. Let $p$ be an isolated point in the topological space $N(X)$. By Lemma 1.15 there exists a sequence $\left\{u_{i}\right\}$ in $T$ such that

$$
\lim _{i \rightarrow \infty} x u_{i}=p=\lim _{i \rightarrow \infty} y u_{i} \text { for } x, y \in X-\{q\},
$$

where $q$ is an exceptional point. Since $p$ is isolated in $N(X)$, we must have that $x u_{i}=y u_{i}=p$, whenever $x, y \in N(X)-\{q\}$ and for $i$ sufficiently large. It follows that $x=y$, whenever $x, y \in N(X)-\{q\}$. The set $N(X)$ accordingly consists of one or two points.

If now $N(X)$ has an isolated point, then according to the previous analysis the set $N(X)$ must be either a fixed point (type la), a union of two fixed points 
(type 2) or an orbit of two elements (type 1d).

If $N(X)$ has no isolated points, then it is a self-dense compact zerodimensional metrizable set and such a set is well known to be a topological Cantor set. The transformation group $(X, T)$ is then of type $1 \mathrm{c}$ if $N(X)$ has a fixed point, and is of type $1 \mathrm{~b}$ if $N(X)$ has no fixed points.

The proof of Theorem 1.7 is completed.

1.17. Theorem. If $(X, T)$ is SAE and if the group $T$ is either connected or abelian, then $N(X)$ is the union of one or two fixed points.

Proof. The theorem is obtained by Gray-Roberson [9] in terms of their stronger hypothesis. The technique of the proof of (1.17) is, however, the same as that of [9]. If $T$ is connected, then each point of the totally disconnected invariant set $N(X)$ must be a fixed point and therefore only type la and type 2 can occur. If $T$ is abelian, we take a point $p \in N(X)$ and apply Lemma 1.15. Let $\left\{u_{i}\right\}$ be the sequence given in (1.15). Take a point $x \in E(X)$ and an abritrary $t \in T$; then $x t \in E(X)$ and by Lemma 1.15 we have

$$
p=\lim _{i \rightarrow \infty}(x t) u_{i}=\lim _{i \rightarrow \infty}\left(x u_{i}\right) t=\left(\lim _{i \rightarrow \infty} x u_{i}\right) t=p t .
$$

Hence $N(X)$ also consists of fixed points for this case and the previous argument applies.

The proof of Theorem 1.17 is completed.

1.18. Remark. If $(X, T)$ is $S A E$, then the phase space $X$ is necessarily separable.

Proof. The property (1.18) is a special case of the following theorem of P. Alexandroff: A connected, locally compact metric space is separable (cf. Pfluger [23, Pp. 22-23]). We give a separate proof for our case.

Let $p \in N(X)$ and $V$ be a compact neighborhood of $p$. Let the sequence $\left\{u_{i}\right\}$ be given as in Lemma 1.15; then by (1.15)

$$
X=\left(\bigcup_{i=1}^{\infty} V u_{i}^{-1}\right) \cup\{q\}
$$

where $q$ is the possible exceptional point. Hence $X$ is a locally compact and $\sigma$-compact metric space, which is therefore separable.

The next property states that SAE are relatively simple if measured by the spread function. In [19] we define the spread function $f_{T}: X \rightarrow[0, \infty]$ by

$$
f_{T}(x)=\inf \left\{\sup \left\{\operatorname{dia}\left(S_{\epsilon}(x) t\right) \mid t \in T\right\} \mid \epsilon>0\right\},
$$

where $S_{\epsilon}(x)$ is the $\epsilon$-open ball around $x$ (compare Remark 1.10). The function $f_{T}$ has the properties that it is upper semicontinuous, $f_{T}(x)=f_{T}(x t)$ for all $x \in X$ 
and $t \in T$ and that $x \in N(X)$ if and only if $f_{T}(x) \neq 0$ (see [20]). In [20, Remark 1.6(3)] we show that if $X$ is a minimal set, then $f_{T}$ is a constant function. For SAE we have one more value for $f_{T}$.

1.19. Remark. If $(X, T)$ is $S A E$, then $f_{T}$ assumes exactly two values.

Proof. By applying Lemma 1.15 to points in $N(X)$ we see that $f_{T}(x)=\operatorname{dia}(X)$ for all $x \in N(X)$. Since $f_{T}(x)=0$ if $x \in E(X)$, the proof is completed.

It should be interesting to give further analysis of the set $N(X)$ of Class II transformation groups of the Introduction by regarding $N(X)$ as a new phase space. We give a simple result of this kind here.

1.20. Remark. If $(X, T)$ is SAE, then the following properties bold:

(1) $N(N(X), T)=\varnothing$ if $(X, T)$ is of types 1a, 2 and $1 \mathrm{~d}$.

(2) $N(N(X), T)=N(X)$ if $(X, T)$ is of types $1 \mathrm{~b}$ and $1 \mathrm{c}$.

Proof. The proof of (1) is obvious. For the proof of (2) we note that $N(X)$ is infinite for those cases. Let $p \in N(X)$. By (1.15) there exist $a, b \in N(X), a \neq b$, and a sequence $\left\{u_{i}\right\}$ in $T$ such that $\lim _{i \rightarrow \infty} a u_{i}=p=\lim _{i \rightarrow \infty} b u_{i}$. It follows that $p \in N(N(X))$.

We have seen that the property of Lemma 1.15, when properly interpreted, gave some interesting dynamical properties. For some other applications we aeed to know the limits $\lim _{i \rightarrow \infty} x u_{i}^{-1}$ for $x \in X$, directed by the inverse sequence $\left\{u_{i}^{-1}\right\}$ in $T$. In this case it would be convenient to limit ourselves to compact phase spaces. If $X$ is not compact, we extend the transformation group to $X^{*}$, the one-point compactification of $X$.

1.21. Lemma. Let $(X, T)$ be a transformation group, where $X$ is compact and let $\left\{t_{n}\right\}$ be a sequence in $T$. Then $\left\{t_{n}\right\}$ bas a nonequicontinuous point in $X$ if and only if $\left\{t_{n}^{-1}\right\}$ bas a nonequicontinuous point in $X$.

Proof. The proof follows directly from some known properties of function spaces (see [8, Chapter 11] and its references). Let $C$ be the set of all continuous maps from $X$ to $X$. If $C$ is given the sup-norm metric, then the function space is complete and its topology coincides with the compact-open topology. Consider the subset $G=\left\{\pi^{t} \mid t \in T\right\}$, the transition group of $T$. Lemma 1.21 follows essentially from the fact that the inverse operation on $G$ is uniformly continuous [8, Theorem 11.18]. Let $A=\left\{\pi^{t} \mid t \in\left\{t_{n}\right\}\right.$. If $A$ is equicontinuous at every point in $X$, the Ascoli theorem shows that $\mathrm{Cl}(A)$, the closure of $A$ in $C$, is compact. Since then the set $\mathrm{Cl}(A)$ is totally bounded, so must be the set $\mathrm{Cl}\left(A^{-1}\right)$, by the uniform continuity of the inverse of $G$. The set $\mathrm{Cl}\left(A^{-1}\right)$ is now totally bounded and complete and is therefore compact. The Ascoli theorem then gives that $A^{-1}$ is equicontinuous at every point of $X$. Hence $\left\{t_{n}\right\}$ is equicontinuous everywhere if and only if $\left\{t_{n}^{-1}\right\}$ is equicontinuous everywhere. 
1.22. Theorem. Let $(X, T)$ be SAE and $X$ be compact. The following properties bold:

(1) For every a $\in N(X)$ there exist some sequence $\left\{s_{i}\right\}$ in $T$ and some $b \in N(X)$ sucb that

$$
\lim _{i \rightarrow \infty} x s_{i}^{-1}=a \text { for all } x \neq b, \quad \lim _{i \rightarrow \infty} x s_{i}=b \text { for all } x \neq a .
$$

(2) The sequence $\left\{s_{i}\right\}$ in (1) can be chosen so that the sequence $\left\{s_{i}\right\}$ the sequence $\left.\left\{s_{i}^{-1}\right\}\right\}$ is nonequicontinuous at $a\{a t b\}$ and equicontinuous elsewbere.

(3) The sequence $\left\{s_{i}\right\}$ in (1) and (2) can be chosen as a suitable subsequence of any sequence which is nonequicontinuous at $a$.

Proof. We begin with a sequence $\left\{s_{i}\right\}$ which is nonequicontinuous at $a$ and modify it successively by subsequences. Step I. Replace $\left\{s_{i}\right\}$ by a subsequence so that every subsequence of this subsequence is nonequicontinuous at $a$. Step II. According to Lemma 1.14 we may replace $\left\{s_{i}\right\}$ by a subsequence again so that $\lim _{i \rightarrow \infty} x s_{i}^{-1}=a$ for all $x \in X-A$, where the set $A$ has at most one element and can be characterized as the set of points where $\left\{s_{i}^{-1}\right\}$ is nonequicontinuous. Since $\left\{s_{i}\right\}$ has a nonequicontinuous point, namely $a$, by Lemma 1.21 the sequence $\left\{s_{i}^{-1}\right\}$ must also have at least one nonequicontinuous point. Hence the set $A$ is a singleton and we denote it as $b$. Step III. Finally we let the sequence $\left\{t_{n}\right\}$ in Lemma 1.14 be $\left\{s_{i}^{-1}\right\}$ and let $p$ be $b$. If we modify $\left\{s_{i}^{-1}\right\}$ by a subsequence which satisfies the conclusion of Lemma 1.14, the final sequence $\left\{s_{i}\right\}$ and $\left\{s_{i}^{-1}\right\}$ have the properties (1), (2) and (3) of Theorem 1.22. The sequence $\left\{s_{i}\right\}$ obtained at the end of Step III is nonequicontinuous at $a$ due to the requirement in Step I; the sequence is nonequicontinuous at $a$ only, due to Lemma 1.12. The sequence $\left\{s_{i}^{-1}\right\}$ obtained at the end of Step III is nonequicontinuous exactly at $b$ due to Lemma 1.21 , which is applied by taking $\left\{t_{n}\right\}$ to be the sequence $\left\{s_{i}\right\}$ at the end of Step III.

The proof of Theorem 1.22 is completed.

We remark that if $(X, T)$ is SAE of type $1 b, 1 c, 1 d$ or 2 , then $X$ is necessarily compact (cf. Theorem 1.28) and so Theorem 1.22 applies. If $(X, T)$ is of the remaining type $1 \mathrm{a}$ and $X$ is not compact, then the property (1) of Theorem 1.22 in general fails (for example take the dilation $f(x)=x / 2$ of the plane and consider the discrete flow generated by the homeomorphism).

Let $(X, T)$ be SAE and suppose that $X$ is noncompact. We will obtain special properties of $N(X)$ by taking the one-point compactification $X^{*}=X \cup\{\infty\}$ of $X$ and by extending the transformation group of $X^{*}$. First we need the following basic property. 
1.23. Proposition. Let $(X, T)$ be a transformation group where $X$ is a locally compact $T_{2}$ uniform space; then $(X, T)$ can be extended (uniquely) to a transformation group $\left(X^{*}, T\right)$.

A straightforward verification gives the proof of Proposition 1.23. Note that it is necessary that $\infty t=\infty$ for all $t \in T$.

If $(X, T)$ is SAE, then by Theorem 1.7 the set $N(X)$ is compact and by Remark 1.18 the space $X$ is separable. According to our earlier remark, Proposition $1.1(5)$, we have that the set $E(X)$ is unchanged if the original metric of $X$ is replaced by one which is induced from $X^{*}=X \cup\{\infty\}$. Hence we have either $N\left(X^{*}\right)=N(X)$ or that $N\left(X^{*}\right)=N(X) \cup\{\infty\}$. For either case it is clear that $N\left(X^{*}\right)$ is zero-dimensional. It is interesting to see that this property holds in more general form. The property given in Remark 1.24 should be important for studying transformation groups of Definition 1.6 with either condition (b) or both conditions (b) and (c) violated.

1.24. Remark. Let $(X, T)$ be a transformation group, where $X$ is a locally compact separable metric space. If $N(X)$ is zero-dimensional, then $N\left(X^{*}\right)$ is zerodimensional. Here an induced metric from $X^{*}$ is used on $X$.

Proof. By Proposition 1.1(3) we have $N\left(X^{*}\right) \subset N(X) \cup\{\infty\}$. Now a zerodimensional space remains zero-dimensional after adding a point (cf. HurewiczWallman [12, p. 19]).

1.25. Lemma. If $(X, T)$ is $S A E$ and if $X$ is noncompact, then $\infty \in N\left(X^{*}\right)$.

Proof. Choose $p \in N(X)$. By Lemma 1.15 there exists a sequence $\left\{u_{i}\right\}$ in $T$ such that $\lim _{i \rightarrow \infty} x u_{i}=p$ for all $x \in X$ except possibly one point. In particular points $x$ arbitrarily near the point $\infty \in X^{*}$ have this property. On the other hand we have $\infty t=\infty$ for all $t \in T$. Hence $\infty \in N\left(X^{*}\right)$.

Now if $(X, T)$ is SAE, then $X^{*}$ is a compact connected metric space and $N\left(X^{*}\right)$ is compact and zero-dimensional and $E\left(X^{*}\right)=E(X)$. It appears that we have already shown that $\left(X^{*}, T\right)$ is SAE. This is not quite the case. The condition that the set $E(X)$ is indivisible by $T$ in $(X, T)$ (Definition $1.6(c)$ ) is not exactly the same as the set $E(X)$ is indivisible by $T$ in $\left(X^{*}, T\right)$. According to Definition 1.4, applied to the present case, the set $E(X)$ is indivisible by $T$ in $\left(X^{*}, T\right)$ if and only if $E(X)$ is indivisible by $T$ in $(X, T)$ and in addition the following property holds:

Property (a). Whenever there exist a sequence $\left\{t_{n}\right\}$ in $T$ and a point $y_{0} \epsilon$ $E(X)$ sucb that $\lim _{n \rightarrow \infty} y_{0} t_{n}=\infty$, then $\lim _{n \rightarrow \infty} y t_{n}=\infty$ for all $y \in E(X)$.

The Property $(\alpha)$ together with the property that $E(X)$ is indivisible by $T$ in $(X, T)$ has been called that $E(X)$ is CC-indivisible by $T$ in $\left(X^{*} T\right)$ [19].

We need a result of [20]. 
1.26. Lemma. Let $(X, T)$ be a transformation group, where $X$ is locally compact. If $x_{0} \in E(X)$ and $\left\{t_{n}\right\}$ is a sequence in $T$ sucb that $\lim _{n \rightarrow \infty} x_{0} t_{n}=p \in N(X)$, then there exists a neighborbood $U$ of $x_{0}$ sucb that if $y \in U$ then the limit points of the sequence $\left\{y t_{n}\right\}$ are all in $N(X)$.

Proof. The lemma is an immediate consequence of Corollary 3.4 in [20], where more generally locally compact $T_{2}$ un iform spaces are considered. The corullary states that the limit points of $\left\{y t_{n}\right\}$ are actually in $F_{u}(X)$, a closed and invariant subset of $N(X)$.

1.27. Theorem. If $(X, T)$ is $S A E$, then $\left(X^{*}, T\right)$ is SAE, provided $X$ is noncompact.

Proof. From the discussion Lemma 1.26, it suffices to prove Property (a). We prove the property by contradiction. Thus assume that there exist a sequence $\left\{t_{n}\right\}$ in $T$ and points $y_{0}, y_{1} \in E(X)$ such that $\lim _{n \rightarrow \infty} y_{0} t_{n}=\infty$ and $\infty$ is not a limit point of the sequence $\left\{y_{1} t_{n}\right\}$. By replacing $\left\{t_{n}\right\}$ with a subsequence we may assume the existence of $z=\lim _{n \rightarrow \infty} y_{1} t_{n}, z \in X$.

We first claim that the sequence $\left\{t_{n}\right\}$ has a nonequicontinuous point in $X$. Thus let $A=\left\{x \in X \mid \lim _{n \rightarrow \infty} x t_{n}=\infty\right\}$. If $\left\{t_{n}\right\}$ is everywhere equicontinuous at $X$, then the set $A$ is relatively closed in $X$. Now if $x_{0} \in A$ then $x_{0} \notin N(X)$, for otherwise the orbit closure of $x_{0}$ is in $X$ and we would not have $\lim _{n \rightarrow \infty} x_{0} t_{n}=\infty$. By applying Lemma 1.26 to the transformation group $\left(X^{*}, T\right)$, we find a neighborhood $U$ of $x_{0}$ such that for each $y \in U$ the limit points of the sequence $\left\{y t_{n}\right\}$ are all in $N\left(X^{*}\right)$. Since $N(X)$ is compact by Theorem 1.7 and since $x_{0} \in E(X)$, we may assume that the limit points of $\left\{y t_{n}\right\}$ are in a neighborhood of $\infty$ disjoint from $N(X)$. It follows that $\lim _{n \rightarrow \infty} y t_{n}=\infty$ for all $y \in U$. Hence the set $A$ is also open in $X$. Since the space $X$ is connected, we have either $A=X$ or $A=\varnothing$. However $A \neq \varnothing$ by our hypothesis in the proof and $A \neq X$, since $X$ contains the compact invariant nonempty set $N(X)$. We then have a contradiction. Hence we may assume that the sequence $\left\{t_{n}\right\}$ has a nonequicontinuous point in $X$.

Let $p \in N(X)$ be a nonequicontinuous point of $\left\{t_{n}\right\}$. It follows from Lemma 1.14 that there exists a subsequence $\left\{s_{k}\right\}$ of $\left\{t_{n}\right\}$ such that $\lim _{k \rightarrow \infty} x s_{k}^{-1}=p$ for all $x \in E(X)$. Let $z \in X$ be the point introduced at the beginning of the proof, i.e. $z=\lim _{n \rightarrow \infty} y_{1} t_{n}$. If $z \in E(X)$, then since $\lim _{k \rightarrow \infty} y_{1} s_{k}=z$, we have $\lim _{k \rightarrow \infty} z s_{k}^{-1}=y_{1}$. Then $y_{1}=p$ and we have a contradiction, since $y_{1} \in E(X)$ and $p \in N(X)$. On the other hand if $z \in N(X)$, then from the hypothesis of $(X, T)$ that $E(X)$ is indivisible by $T$ in $(X, T)$, we have that $\lim _{n \rightarrow \infty} y t_{n}=z$ for all $y \in E(X)$. This, however, is impossible, since $\lim _{n \rightarrow \infty} y_{0} t_{n}=\infty$ and $y_{0} \in E(X)$. We then have a contradiction for the case that $\left\{t_{n}\right\}$ has a nonequicontinuous point in $X$. 
The proof of Theorem 1.27 is completed. Note that the proof can be shortened in case $E(X)$ is known to be connected.

As a consequence of Theorem 1.27, we have the following important property for almost equicontinuous transformation groups.

1.28. Theorem. If $(X, T)$ is SAE (Definition 1.6) and if $X$ is not compart, then $N(X)$ is a fixed point.

Proof. It follows from Theorem 1.27 that $\left(X^{*}, T\right)$ is SAE. By Lemma 1.25 we have $N\left(X^{*}\right)=N(X) \cup\{\infty\}$. By applying Theorem 1.13 on $\left(X^{*}, T\right)$ we see that $N(X)$ is a fixed point.

1.29. Corollary. Let $(X, T)$ be SAE such that $N(X)$ is a union of two fixed points $p$ and $q$. Then there exists a sequence $\left\{s_{i}\right\}$ in $T$ such that

$$
\lim _{i \rightarrow \infty} x s_{i}^{-1}=p \text { for all } x \neq q, \quad \lim _{i \rightarrow \infty} x s_{i}=q \text { for all } x \neq p \text {. }
$$

Proof. By Theorem 1.28 the space $X$ is compact. In Theorem 1.22 we have such a sequence $\left\{s_{i}\right\}$ for two points $a, b \in N(X)$, which are not necessarily distinct. The fact that $p$ and $q$ are fixed points gives that they are distinct and $\{a, b\}=\{p, q\}$.

1.30. Example. (1) The discrete flows generated by homeomorphisms which are the usual linear fractional transformations on the 2-sphere give examples of type 2 and type la of Theorem 1.7 (see Kerékjártó [16] and Theorem 1.8). The examples can easily be extended to higher dimensional spheres and to continuous flows.

(2) Let $T$ be the discrete free group of two generators $s$ and $t$ acting on the 2-sphere $X$, where $t$ acts as the extension of the dilation of the plane to the sphere by adding the fixed point $\{\infty\}$ and $s$ is the reflection with respect to the equator. It is readily verified that $(X, T)$ is of type $1 \mathrm{~d}$. Here $N(X)$ is the union of the north pole and the south pole of the 2-sphere, and $\infty=$ north pole.

(3) We have no examples for types $1 b$ and $1 c$. However, it seems that construction similar to (2) may yield such examples.(1)

We remark that in the study of $(X, T)$ of Definition 1.6 and related classes of transformation groups the condition that $X$ is connected can sometimes be omitted. For example if $T$ is connected, then each component of $X$ is invariant and the investigation may be reduced to the components of $X$.

(1) Such examples have recently been found. They will appear in a forthcoming paper. 
2. The set $N(X)$, arbitrary dimension. In $\$ 1$ we have seen a relatively complete analysis of transformation groups whose set of nonequicontinuous points $N(X)$ is zero-dimensional. In particular, it is shown in Theorem 1.7 that for a large class of such transformation groups the set $N(X)$ is of special topological nature. Presumably this phenomenon holds without the assumption that $N(X)$ is of zerodimension. As a beginning step we will apply results of $\$ 1$ to more general cases by making quotient structures. We will make decomposition $X_{*}$ of $X$ so that the acting group $T$ permutes members of $X_{*}$ and that $X_{*}$, endowed with the usual quotient topology, becomes metrizable and the canonically induced quotient transformation group $\left(X_{*}, T\right)$ is SAE (strictly almost equicontinuous). In this way we obtain results on the set of components of the set $N(X)$, or more generally, of an arbitrary invariant subset $S$ which contains $N(X)$.

The main result which we obtain in this section is Theorem 6 of [19] which is here generalized in several ways. The compactness hypothesis of the set $N(X)$ in [19] is replaced by the more general property that $N(X)$ is semiclosed in the sense of Whyburn [25]. The set $N(X)$ is further replaced by an arbitrary semiclosed invariant subset which contains it. The various topological hypotheses in Theorem 6 of [19] are replaced by a single condition of indivisibility in Theorem 2.12. An analogue theorem of [19, Theorem 6] for noncompact phase spaces is obtained, which shows that in this case $N(X)$ is a continuum (cf. Theorem 2.17).

Now associated with the decomposition $X_{*}$ of $X$ there is a canonical projection $\pi: X \rightarrow X_{*}$ (onto). This projection is a transformation group homomorphism, i.e., $\pi$ is continuous and $\pi(x t)=(\pi(x)) t$ for all $x \in X, t \in T$. In general the image of an equicontinuous point of $x \in X$ under $\pi$ is not necessarily an equicontinuous point of $X_{*}$. However, it is true for a useful case as given in the following remark. For more discussion of effects of homomorphisms on equicontinuous points see our forthcoming work on dynamical properties of almost equicontinuity.

2.1. Proposition. Let $\left(X_{i}, T\right)$ be transformation groups, where $X_{i}$ are metric spaces with fixed metrics $(i=1,2)$. Let $f$ be a homomorphism of $\left(X_{1}, T\right)$ onto $\left(X_{2}, T\right)$ and $x_{0} \in E\left(X_{1}\right)$. If $f$ is uniformly continuous and there exists a base $\left\{B_{n} \mid n=1,2, \ldots\right\}$ of neighborboods at $x_{0}$ such that $f\left(B_{n}\right)$ are neigbborboods of $f\left(x_{0}\right)$ for each $n=1,2, \ldots$. Then $f\left(x_{0}\right) \in E\left(X_{2}\right)$.

Proof. Let $\epsilon>0$ be given. By the uniform continuity of $f$ there exists $\delta_{1}>0$ such that $x, y \in X_{1}$ and $d_{1}(x, y)<\delta_{1}$ imply that $d_{2}(f(x), f(y))<\epsilon$, where $d_{i}$ are the metrics on $X_{i}(i=1,2)$. By the fact that $x_{0} \in E(X)$ we have a $\delta_{2}>0$ such that $y \in X_{1}$ and $d_{1}\left(x_{0}, y\right)<\delta_{2}$ imply that $d_{1}\left(x_{0} t, y t\right)<\delta_{1}$ for all $t \in T$. Choose $B$ to be one of the $\left\{B_{n}\right\}$ such that $d_{1}\left(x_{0}, b\right)<\delta_{2}$ for all $b \in B$. By 
hypothesis $f(B)$ is a neighborhood of $f\left(x_{0}\right)$. There exists a $\delta_{3}>0$ such that the $\delta_{3}$-open ball of $f\left(x_{0}\right)$ is in $f(B)$. Now if $u \in X_{2}$ and $d_{2}\left(u, f\left(x_{0}\right)\right)<\delta_{3}$ then we claim $d_{2}\left(u t, f\left(x_{0}\right) t\right)<\epsilon$ for all $t \in T$. It would follow from the claim that $f\left(x_{0}\right) \in E\left(X_{2}\right)$.

To see the claim we let $b \in B$ be such that $f(b)=u$. Then $d_{1}\left(x_{0}, b\right)$ $<\delta_{2}$ : hence $d_{1}\left(x_{0} t, b t\right)<\delta_{1}$ for all $t \in T$. Hence for all $t \in T$ we have $d_{2}\left(f\left(x_{0} t\right), f(b t)\right)<\epsilon$. Since $f\left(x_{0} t\right)=f\left(x_{0}\right) t, f(b t)=f(b)_{t}=u t$, we have $d_{2}\left(u t, f\left(x_{0}\right) t\right)<\epsilon$ for all $t \in T$.

The proof of Proposition 2.1 is completed.

A similar proof shows that Proposition 2.1 holds for uniform $T_{2}$ phase spaces $X_{1}$ and $X_{2}$.

Note that it is a corollary of the uniform space version of Proposition 2.1 that if $(X, T)$ is a transformation group, with $X$ a locally compact $T_{2}$ uniform space and if we take the one-point compactification transformation group $\left(X^{*}, T\right)$ then $E(X) \subset E\left(X^{*}\right)$. This is because the inclusion map $i: X \rightarrow X^{*}$ is uniformly continuous. This result was proved directly in [20]. The result was given as Proposition $1.13(3)$ in [20].

In defining the class of transformation groups SAE of $\$ 1$ (Definition 1.6) we require that the set $E(X)$ be indivisible by $T$. Similarly in studying an arbitrary $N(X)$, not necessarily zero-dimensional, or an invariant set $S$ which contains $N(X)$ it requires a suitable condition of indivisibility on the set $E(X)=X-N(X)$, or, respectively, on the set $X-S$. Such a general setting of indivisibility is provided in [20]. The following is a special indivisibility which we will need.

2.2. Definition. Let $(X, T)$ be a transformation group and $Y \subset X$. The set $Y$ is said to be indivisible by $T$ if for every sequence $\left\{t_{n}\right\}$ in $T$ whenever there exists some $y_{0} \in Y$ such that the sequence $\left\{y_{0} t_{n}\right\}$ has a limit point in a component $K$ of $N(X)$, then for every $y \in Y$ the sequence $\left\{y t_{n}\right\}$ has a limit point in $K$.

Definition 2.2 extends Definition 1.4, where $N(X)$ is required to be zerodimensional. Definition 1.4 is supported by topological sufficient conditions given in Theorem 1.5. Likewise the present definition is supported by the following two theorems.

2.3. Theorem. Suppose that $(X, T)$ is a transformation group, $X$ is a locally compact metric space and that the metric on $X$ is complete. A sufficient condition for a connected subset $Y$ of $E(X)$ to be indivisible ly $T$ is that one of the following cases occurs.

(1) $Y \cap \mathrm{Cl}(N(X))=\varnothing$.

(2) $Y$ is locally compact and invariant.

(3) $N(X)$ is scattered.

Moreover, if $N(X)$ is either zero-dimensional or $\mathrm{Cl}(N(X))$ is compact the completeness condition of the metric is not needed. 
Proof. The theorem follows from Theorem 4.1 of [20]. In [20, Theorem 4.1] the problem is treated somewhat differently, where instead of a metric space $X$ is assumed to be a uniform $T_{2}$ space and instead of concluding on the property of Definition 2.2 it concludes on a slightly stronger condition that $Y$ is completely indivisible by the class of all nets $Q$ in $T, Q \pitchfork Y$ (cf. [20, Definition 2.2, 3.1]).

2.4. Theorem. Suppose that $(X, T)$ is a transformation group, $X$ is a locally compact metric space and the components of $N(X)$ are compact. A sufficient condition for a connected subset $Y$ of $E(X)$ to be indivisible by $T$ is that one of the following cases occurs:

(1) $Y$ is a semicontinuum.

(2) Either $X$ or $Y$ is locally connected.

(3) $N(X)$ is locally compact.

Moreover the set $Y$ in conditions (1) and (2) may be replaced by one of its dense subsets.

Proof. The theorem follows from Theorem 4.2 of [20]. The remark given in the proof of Theorem 2.3 also applies here.

Out of the six conditions given in the two previous theorems we select two important applications for illustration.

2.5. Corollary. Suppose that $(X, T)$ is a transformation group, where $X$ is a locally compact metric space. Then a connected subset $Y$ of $E(X)$ is indivisible by $T$ provided either one of the following conditions bolds:

(1) The set $N(X)$ is compact.

(2) The set $N(X)$ bas compact components and the space $X$ is locally connected.

Proof. (1) follows from condition (1) and the last remark of Theorem 2.3. (2) follows from condition (2) of Theorem 2.4.

We now come to the choice of topological hypothesis of the set $N(X)$ or most generally of the set $S$ which contains it. We will use the hypothesis that the set $S$ is semiclosed in the sense of Whyburn [25, p. 131]. A subset $K$ of a metric space $X$ is said to be semiclosed if its components are closed and any convergent sequence of components of $K$ whose limit set intersects $X-K$ converges to a single point of $X-K$. If the space $X$ is compact, this is equivalent to the condition that for every $\epsilon>0$ the union of all the components of $K$ of diameter $\operatorname{dia}(K) \geq \epsilon$ is a closed set [25, p. 132, (5.4)]. We prefer the semiclosed condition to the more restricted one that $S$ is compact. The replacement of the semiclosed condition of $S$ in Definition 2,6 by compactness will slightly simplify a few proofs in this section, but it would lack the generality to include sets such as arbitrary zero-dimensional sets. 
2.6. Definition. Let $(X, T)$ be a transformation group, where $X$ is a compact metric space. Let $S$ be a nonempty, semiclosed, invariant subset of $X$. We let $X_{*}(S)$ denote the decomposition of $X$, whose equivalence classes are components of $S$ and points of $X-S$. Let $X_{*}(S)$ be given the quotient topology. Then canonically $(X, T)$ induces a transformation group $\left(X_{*}(S), T\right)$. Let $\pi: X \rightarrow X_{*}(S)$ denote the canonical projection which takes every point of $X$ to its equivalence class. The action of $\left(X_{*}(S), T\right)$ is determined by the law that $\pi(x t)=(\pi(x)) t$ for $x \in X, t \in T$, i.e. by requiring that $\pi$ is a homomorphism of $(X, T)$ onto $\left(X_{*}(S), T\right)$. We call $\left(X_{*}(S), T\right)$ the $S$-partition transformation group of $(X, T)$ and the canonical map $\pi$ the $S$-projection of $(X, T)$.

According to a theorem in Whybum [25, Chapter VII, (5.1), (5.2)] a necessary and sufficient condition that $X_{*}(S)$ is metrizable is that $S$ is semiclosed. Hence $X_{*}(S)$ of Definition 2.6 is a compact metric space. The decomposition $X_{*}(S)$ of $X$ is upper semicontinuous in this case.

The case when $X$ is noncompact will be considered later in the section.

2.7. Proposition. Let $\left(X_{*}(S), T\right)$ be the S-partition transformation group of Definition 2.6. The following properties bold:

(1) The S-projection $\pi$ is a transformation group bomomorpbism of $(X, T)$ onto $\left(X_{*}(S), T\right)$.

(2) The restriction of $\pi$ to $X-S$ is a bomeomorphism onto its image.

(3) If $x \in X-S$, then there exists a decreasing sequence of neighborboods $\left\{B_{n}\right\}$ of $x$ such that $\left\{\pi\left(B_{n}\right)\right\}$ are neigbborboods of $\pi(x)$.

(4) If $x \in E(X) \cap(X-S)$ then $\pi(x) \in E\left(X_{*}(S)\right)$.

Proof. (1) and (2) follow immediately from the definition of $\left(X_{*}(S), T\right)$.

(3) If $x \in X-S$ then $\pi^{-1}(\pi(x))=x$. Let $\left\{U_{n}\right\}$ be a decreasing sequence of compact neighborhoods of $\pi(x)$ such that $\pi(x)=\bigcap_{n} U_{n}$. Then

$$
x=\pi^{-1}(\pi(x))=\pi^{-1}\left(\bigcap_{n} U_{n}\right)=\bigcap_{n} \pi^{-1}\left(U_{n}\right) .
$$

Hence $\left\{\pi^{-1}\left(U_{n}\right)\right\}$ form a base of neighborhoods of $x$ whose images under $\pi$ are neighborhoods of $\pi(x)$.

(4) follows from (3) and Proposition 2.1.

The proof of Proposition 2.7 is completed.

2.8. Lemma. Let $\left(X_{*}(S), T\right)$ be the S-partition transformation group of Definition 2.6. If $X-S$ bas a point which is not almost periodic, then $N\left(X_{*}(s)\right) \neq \varnothing$.

Proof. By (1) and (2) of Proposition 2.7 the restriction of $\pi$ to $X-S$ is a transformation group isomorphism of $(X-S, T)$ onto $(\pi(X-S), T)$. Hence $X_{*}(S)$ 
also has a point which is not almost periodic. If $N\left(X_{*}(S)\right)$ were empty then $\left(X_{*}(S), T\right)$ is a uniformly equicontinuous transformation group. According to Gottschalk-Hedlund [8, Theorem 4.38] such a transformation group is uniformly almost periodic. We would then have a contradiction. Hence $N\left(X_{*}(S)\right) \neq \varnothing$ as asserted in the lemma.

2.9. Lemma. Let $\left(X_{*}(S), T\right)^{\prime}$ be the S-partition transformation group of Definition 2.6. If $N(X) \subset S$ then $N\left(X_{*}(S)\right)$ is totally disconnected.

Proof. Let $\pi$ be the S-projection of Definition 2.6. If $N(X) \subset S$, then $E(X) \cap$ $(X-S)=X-S$. According to Proposition 2.7(4) we must then have $N\left(X_{*}(S)\right) \subset$ $\pi(S)$. By the topology given to $X_{*}(S)$ the set $\pi(S)$ is totally disconnected, so must be the subset $N\left(X_{*}(S)\right)$.

In order to verify a condition on indivisibility of the next theorem, we need the following lemma, which follows from two results of [20].

2.10. Lemma. Let $(X, T)$ be a transformation group, where $X$ is a locally compact separable metric space. Let $Y$ be a subset of $E(X)$ and $D$ be a dense subset of $Y$. If the components of $N(X)$ are compact, then $Y$ is indivisible by $T$ if and only if $D$ is indivisible by $T$.

Proof. The lemma is a consequence of the general result [20, Lemma 3.7]. Lemma 3.7 of [20] shows that Lemma 2.10 holds for a locally compact $T_{2}$ uniform space and for the kind of indivisibility that is given in Definition 2.2, except that all sequences there are to be replaced by nets. For locally compact separable metric spaces Definition 2.2 and its analogue with sequences to be replaced by nets are equivalent according to [20, Proposition 2.9].

The proof of Lemma 2.10 is completed.

A major theorem follows.

2.11. Theorem. Suppose that $\left(X_{*}(S), T\right)$ is the S-partition transformation group of Definition 2.6, constructed out of a transformation group $(X, T)$ and a semiclosed subset $S$ of $X$, where $X$ is now assumed to be connected as well as compact. Suppose also that the following conditions are satisfied:

(1) $N(X) \subset S$.

(2) The set $X-S$ is indivisible by $T$ (Definition 2.2).

(3) There exist some $x \in X-S$ which is not almost periodic. Then $\left(X_{*}(S), T\right)$ is SAE (Definition 1.6).

Proof. Clearly $X_{*}(S)$ is a compact connected metric space. It remains to verify conditions (a), (b), and (c) of Definition 1.6 for the transformation group $\left(X_{*}(S), T\right)$. In order to satisfy condition (a) the set $N\left(X_{*}(S)\right)$ must be nonempty and totally disconnected. Since, by hypothesis (3) of the theorem, the set $X-S$ 
has a point which is not almost periodic, the set $N\left(X_{*}(S)\right)$ is nonempty according to Lemma 2.8. Since, by hypothesis (1) of the theorem, $N(X) \subset S$, we must have that $N\left(X_{*}(S)\right)$ is totally disconnected (use Lemma 2.9). Thus condition (a) has been verified. Condition (b) of (1.6) is automatically satisfied, since the space $X_{*}(S)$ is compact.

It remains to verify $(c)$ of $(1.6)$ for $\left(X_{*}(S), T\right)$. Since $\pi(S)$ is totally disconnected and $X_{*}(S)$ is connected, the set $\pi(X-S)$ is dense in $X_{*}(S)$. The set $\pi(X-S)$ is a subset of $E\left(X_{*}(S)\right)$ by Proposition 2.7(4). By Lemma 2.10 it then suffices to show that the set $Y=\pi(X-S)$ is indivisible by $T$. According to Definition 1.4 to show the latter is to show that for every sequence $\left\{t_{n}\right\}$ in $T$ if there exists a point $y_{0} \in Y$ such that $\lim _{n \rightarrow \infty} y_{0} t_{n}=p \in N\left(X_{*}(S)\right)$, then the sequence $\left\{y t_{n}\right\}$ has $p$ as a limit point for all $y \in Y$. We let $x$ and $x_{0}$ be points in $X-S$ such that $\pi(x)=y$ and $\pi\left(x_{0}\right)=y_{0}$. By the compactness of $X$ the sequence $\left\{x_{0} t_{n}\right\}$ has a limit point $z \in X$. It follows from the equation $\pi(a t)=\pi(a) t$ for all $a \in X, t \in T$, that $\pi(z)=p$. Since $\pi(X-S) \subset E\left(X_{*}(S)\right)$, we have $\pi^{-1}\left[N\left(X_{*}(S)\right)\right] \subset S$. Hence $z \in S$. Since $N(X)$ may be a proper subset of $S$, the point $z$ is conceivably a point of $E(X)$. We show that this cannot occur. For if $z \in E(X)$ then $x_{0}$ would be a limit point of $\left\{z t_{n}^{-1}\right\}$. It would follow that $y_{0}$ is a limit point of $\left\{p t_{n}^{-1}\right\}$. This is impossible, since $p$ is in $N\left(X_{*}(S)\right)$, so must be any point in its orbit closure. We conclude that $z \in N(X)$. Let $K$ be the component of $N(X)$ which contains $z$. Then the sequence $\left\{x_{0} t_{n}\right\}$ has a limit point in $K$. Since the set $X-S$ is assumed to be indivisible by $T$ in hypothesis (2), we must have that $\left\{x t_{n}\right\}$ also has a limit point in $K$ (cf. Definition 2.2). By projecting down to $X_{*}(S)$ by the map $\pi$, it follows that the sequence $\left\{y t_{n}\right\}$ has a limit point in $\pi(K)$. Since the set $N(X)$ is a subset of $S$, the component $K$ of $N(X)$ lies in a component $L$ of $S$. Then $L \in X_{*}(S)$. Since $z \in K$ and $\pi(z)=p$, we conclude that $\pi(K)=p$. Hence $\left\{y t_{n}\right\}$ has a limit point in $p$. This proves (c) of Definition 1.6 for $\left(X_{*}(S), T\right)$.

The proof of Theorem 2.11 is completed.

The following analysis of the set $S$ can be readily derived by projecting $X$ to $X_{*}(S)$ and by applying Lemma 1.15 to $\left(X_{*}(S), T\right)$. The set $S$ of Theorem 2.11 splits into two disjoint sets $A$ and $B$. The set $A=\pi^{-1}\left[N\left(X_{*}(S)\right)\right]$ is compact invariant and is precisely the union of those components $C$ of $S$ which have the following property: There exist $p \in C, x \in X-S$ and sequence $\left\{t_{n}\right\}$ in $T$ such that $\lim _{n \rightarrow \infty} x t_{n}=p \in C$. Each component of $A$ then contains a component of $N(X)$. The set $B=S-A$ is invariant and is a union of components of $S$. The set $B \cap N(X)$ is then the union of all components of $N(X)$ which lie in $B$. If $x \in X-S$, and $p \in B$ and $\left\{t_{n}\right\}$ a sequence in $T$ then $\lim _{n \rightarrow \infty} x t_{n} \neq p$.

A number of similar applications of Theorem 2.11 can be obtained. The following theorem gives some of the important consequences. 
2.12. Theorem. Let $(X, T)$ be a transformation group, where $X$ is a compact connected metric space. Suppose that the set $N(X)$ is semiclosed and nonempty, the set $E(X)$ is indivisible by $T$ and that there exists some $x \in E(X)$ which is not almost periodic. Then the following properties bold:

(1) $N(X)$ bas at most two invariant components.

(2) If $T$ is connected, then $N(X)$ bas exactly one or two components; in particular the set $N(X)$ is compact.

(3) If $T$ is abelian, then $N(X)$ bas at least one invariant component.

(4) If $N(X)$ bas finite number of components, then the number is at most two and $N(X)$ is the union of those two components.

(5) The set of periodic points in $(X, T)$ is contained in two (possibly one) components of $N(X)$.

Proof. We let $S=N(X)$ and form the $S$-partition transformation group $\left(X_{*}(S), T\right)$ as defined in Definition 2.6. Let $\pi: X \rightarrow X_{*}(S)$ be the $S$-projection. We observe that if $K$ is a component of $N(X)$, then $K$ is invariant if and only if $\pi(K)$ is a fixed point of $X_{*}(S)$. Another observation is that, whether $X$ is compact or not, if $P$ is a finite invariant subset of $X$ and $(X, T)$ is SAE, then $P C$ $N(X)$. This fact follows, for instance, from Lemma 1.15. It follows in turn from Theorem 1.7 that $P$ has at most two points. These observations prove (1) and (4).

For proving (2) it suffices to see that every component of $N(X)$ under a connected group is invariant.

(3) If $T$ is abelian, then $N\left(X_{*}(S)\right)$ has a fixed point $y$ by Theorem 1.17. The set $\pi^{-1}(y)$ is then necessarily an invariant component of $N(X)$.

(5) If $x \in X$ is periodic, then $x T$ is compact and invariant. Then $\pi(x) T$ is a compact orbit in $\left(X_{*}(S), T\right)$. By Lemma 1.15 the only compact orbits of $\left(X_{*}(S), T\right)$ are those in $N\left(X_{*}(S)\right)$. An examination of Theorem 1.7 shows that the only compact orbits in $N\left(X_{*}(S)\right)$ are either fixed points (type 2, 1a or 1c) or an orbit of two points (type 1d). The proof of ( 5 ) then follows.

2.13. Remark. Theorem 2.12 bolds if $N(X)$ and $E(X)$ are replaced by $S_{0}$ and $X-S_{0}$, where $S_{0}$ is any semiclosed invariant set which contains $N(X)$. Moreover, eacb invariant component of $S_{0}$ contains a component of $N(X)$.

Proof. Save for the last statement, the proof is the same as that of (2.12), with $N(X)$ and $E(X)$ to be replaced by $S_{0}$ and $X-S_{0}$. The last statement comes from the fact that such a component $C$ is expressible as $C=\pi^{-1}(y), y$ a fixed point of $N\left(X_{*}\left(S_{0}\right)\right)$. In the notation of the analysis following Theorem 2.11 the set $C$ is then a component of the set $A$. Hence $C$ contains a component of $N(X)$.

Perhaps we should point out that we prefer the general set $S$ to the special set $N(X)$ in Definition 2.6 and Theorem 2.11 for reasons more than just generality. In general the set $N(X)$ is not semiclosed, but there are interesting semiclosed 
sets which contain $N(X)$, for instance, the set $\mathrm{Cl}(N(X))$. There are also cases like in the proof of Theorem 3.4(3), where the $N(X)$ is zero-dimensional, whence it is semiclosed, but it is the boundary components (of the manifold) which contain $N(X)$ that we want to determine.

It is shown in (2) of Theorem 2.12 that under the hypothesis of the theorem if $T$ is a connected group, then $N(X)$ has at most two components. A result of this kind is now given for arbitrary acting group.

2.14. Lemma. Let $(X, T)$ be a transformation group, where $X$ is a compact connected metric space. Suppose a subset $S$ of $X$ bas the following properties:

(a) $S$ is a nonempty, semiclosed and invariant set which contains $N(X)$.

(b) The set $X-S$ is indivisible by $T$.

(c) There exists some $x \in X-S$ which is not almost periodic.

Let $x_{1}, x_{2} \in S$. If there is no component of $S$ which meets both $\mathrm{Cl}\left(x_{1} T\right)$, and $\mathrm{Cl}\left(x_{2} T\right)$, then $\mathrm{Cl}\left(x_{i} T\right)$ lies in a component $K_{i}$ of $S(i=1,2)$.

Proof. Let $\left(X_{*}(S), T\right)$ be again the $S$-partition transformation group of Definition 2.6 and $\pi$ be the $S$-projection. Let $A_{i}=\mathrm{Cl}\left(x_{i} T\right)(i=1,2)$. Since no component of $S$ intersects both $A_{1}$ and $A_{2}$, we have $\pi\left(A_{1}\right) \cap \pi\left(A_{2}\right)=\varnothing$. If $y_{i}=$ $\pi\left(x_{i}\right)$, then $\pi\left(A_{i}\right)=\mathrm{Cl}\left(y_{i} T\right)(i=1,2)$. Hence $\pi\left(A_{1}\right)$ and $\pi\left(A_{2}\right)$ are two disjoint orbit closures in the transformation group $\left(X_{*}(S), T\right)$ which is SAE. We now apply Theorem 1.22(1). The transformation group $(X, T)$ in (1.22) is now replaced by $\left(X_{*}(S), T\right)$, and the point $a$ there is now taken to be $\pi\left(x_{1}\right)$. The first equation Theorem 1.22 then implies that the entire orbit closure $\pi\left(A_{2}\right)$ must degenerate to the single point $b$. Hence $\pi\left(A_{2}\right)$ is a fixed point $b$. Likewise $\pi\left(A_{1}\right)$ is a fixed point $a$. Let $K_{i}(i=1,2)$ be components of $S$ defined by $K_{1}=\pi^{-1}(a)$ and $K_{2}=\pi^{-1}(b)$. Then $\mathrm{Cl}\left(x_{i} T\right)=A_{i} \subset K_{i}(i=1,2)$.

The proof of the lemma is completed.

2.15. Definition. Let $(X, T)$ be a transformation group and let $Y$ be an invariant subset of $X$. A dynamical separation of $Y$ is defined to be a decomposition of $Y$ into disjoint nonempty invariant subsets $K$ and $L$ such that neither set has a limit point of the other. If such a separation exists, the set $Y$ is called dynamically disconnected (such a set is also called decomposable by Smale [24]).

2.16. Theorem. Let $(X, T)$ be a transformation group, where $X$ is a compact connected metric space. Let $S$ be a subset of $X$ which bas the properties (a), (b) and (c) of Lemma 2.14. If $S$ is dynamically disconnected, then $S$ is the union of two invariant components.

Proof. We let $S=K \cup L$ be a dynamical separation of $S$. Let $x \in K$ and $y \in L$. Then $x T \subset K$ and $y T \subset L$, since $K$ and $L$ are invariant. Since neither one of $K$ and $L$ has a limit point of the other set, we have $\operatorname{Cl}(x T) \cap L=\varnothing$ and 
$\mathrm{Cl}(y T) \cap K=\varnothing$. Now if $C$ is a component of $S$, then $C$ cannot simultaneously meet $K$ and $L$, whence it cannot meet both $\mathrm{Cl}(x T)$ and $\mathrm{Cl}(y T)$. By Lemma 2.14, $x$ is contained in an invariant component of $S$ in $K$ and $y$ is contained in an invariant component of $S$ in $L$. It follows that $K$ and $L$ are the union of invariant components of $S$. By Remark 2.13 we have that Theorem 2.12(1) holds if $N(X)$ is replaced by $S$ and $E(X)$ is replaced by $X-S$. It follows that $K$ is an invariant component of $S$ and $L$ is an invariant component of $S$.

The proof of Theorem 2.16 is completed.

Up to now $\$ 2$ considers only transformation groups which have compact phase spaces. We now give results for those whose spaces are noncompact. The results are given in Theorem 2.17 and Corollary 2.18.

2.17. Theorem. Let $(X, T)$ be a transformation group, where $X$ is a locally compact connected metric space which is noncompact. Let $S$ be a nonempty subset of $X$ which bas the following properties:

(a) The components of $S$ are compact.

(b) Any convergent sequence of components of $S$ whose limit set intersects $X-S$ converges to a single point of $X-S$.

(c) $\mathrm{Cl}(p T)$ is compact for all $p \in S$.

(d) The set $X-S$ is indivisible by $T$.

(e) There exists $x \in X-S$ which is not almost periodic.

(f) $N(X) \subset S$.

Then $S$ is a continuum.

Proof. First by the theorem of P. Alexandroff that a locally compact connected $T_{2}$ metric space is separable (cf. [23]) we have that $X$ is separable. We let $Y$ be the quotient space of $X$ whose equivalence classes are components of $S$ and points of $X-S$. The decomposition $Y$ is upper semicontinuous; hence $Y$ is metrizable and $(X, T)$ induces canonically a transformation group $(Y, T)$ such that $\phi(x t)=(\phi(x)) t$ for all $x \in X, t \in T$, where $\phi: X \rightarrow Y$ is the canonical projection which takes every point to its equivalence class. To prove those properties rigorously one may take the one-point compactification transformation group $\left(X^{*}, T\right)$ and then take the $S$-partition transformation group $\left(\left(X^{*}\right)_{*}(S), T\right)$ of Definition 2.6. Note that from (a) and (b) the set $S$ is given as semiclosed in $X$. It is also semiclosed in $X^{*}$. To show the latter it suffices to see that if a sequence of components of $S$ converges and has $\infty$ as one of the limit points, then $\infty$ is the only limit point. This is true, since in a compact metric space the limit of a convergent sequence of a connected set is connected. Note that if $\pi$ is the S-projection of $\left(X^{*}, T\right)$ to $\left(\left(X^{*}\right)_{*}(S), T\right)$ then $Y=\left(X^{*}\right)_{*}(S)-\pi(\infty)$. This argument also shows that if $X$ is given the induced metric from $X^{*}$ and $Y$ is given the induced metric from $Y^{*}=\left(X^{*}\right)_{*}(S)$, then $\phi$ is uniformly continuous. Note that $\phi=\left.\pi\right|_{X}$ 
We now give $X$ and $Y$ induced metrics from their one-point compactifications. This does not change the set $N(X)$ according to Proposition 1.1(5). If $x \in$ $E(X) \cap(X-S)$ then $\phi(x) \in E(Y)$. The proof is the same as that of Proposition 2.7(4). Since $N(X) \subset S$, we have $E(X) \supset X-S$. Hence $\phi(X-S) \subset E(Y)$. We claim that $(Y, T)$ is SAE.

Condition (b) of Definition 1.6 is due to (c) here and that $N(Y) \subset \phi(S)$. (a) of (1.6) is satisfied. That $N(Y) \neq \varnothing$ is obtained by showing $N\left(Y^{*}\right) \neq \varnothing$ (use (2.8)) and that $\pi(\infty) \neq N\left(Y^{*}\right)$ (use (1.15)). For (c) of (1.6) we need to show that $E(Y)$ is indivisible by $T$. The set $\phi(X-S)$ is dense in $Y$ according to the definition of $Y$. By Lemma $2.10, E(Y)$ is indivisible by $T$ if and only if $\phi(X-S)$ is indivisible by $T$. That $\phi(X-S)$ is indivisible by $T$ is a consequence of the fact that $X-S$ is indivisible by $T$. This is argued the same as in the proof of Theorem 2.11. Hence $(Y, T)$ is SAE. Since $Y$ is noncompact, by Theorem 1.28 the set $N(Y)$ is a fixed point $p$. Let $\infty_{1}$ and $\infty_{2}$ be points at infinity of $X$ and $Y$ respectively. If we apply Corollary 1.29 to $\left(Y^{*}, T\right)$ we find a sequence $\left\{s_{i}\right\}$ in $T$ such that

$$
\lim _{i \rightarrow \infty} y s_{i}^{-1}=p \text { for all } y \in Y, \quad \lim _{i \rightarrow \infty} y s_{i}=\infty_{2} \text { for all } y \in Y-\{p\} .
$$

Let $x \in X-\phi^{-1}(p)$; then $t=\phi(x) \neq p$; hence we must have $\lim _{i \rightarrow \infty} x s_{i}=\infty_{1}$. Since points in $S$ have compact orbit closure, we then have $S \subset \phi^{-1}(p)$. Since $\phi^{-1}(p)=\phi^{-1}(N(Y)) \subset S$, we conclude that $S=\phi^{-1}(p)$. Hence $S$ is its own component and is therefore compact and connected.

The proof of Theorem 2.17 is completed.

We give a special application of Theorem 2.17.

2.18. Corollary. Let $(X, T)$ be a transformation group, where $X$ is locally compact, connected and noncompact. Let $E(X)$ be connected and contain a point which is not almost periodic. If $N(X)$ is compact, then it is a continuum.

Proof. We verify that $(X, T)$ satisfies the conditions of Theorem 2.17, with $S=N(X)$. Condition (f) is automatically satisfied. Condition (e) is satisfied, since $X-S=E(X)$. Conditions (a), (b) and (c) are satisfied since $N(X)$ is compact. Finally condition (d), which is the same as that $E(X)$ is indivisible by $T$, is satisfied by Corollary $2.5(1)$.

In Theorems $2.11,2.12,2.16,2.17$ and Corollary 2.18 we require the condition that $E(X)$ has a point which is not almost periodic. It would then be desirable to give sufficient conditions for this condition. We give them in the following two propositions.

2.19. Proposition. Let $(X, T)$ be a transformation group. In order that $E(X)$ has a point which is not almost periodic it is sufficient, and in case $X$ is compact, also necessary, that the following property bold: 
(i) There exist points $p \in N(X), x_{0} \in E(X)$ and a sequence $\left\{t_{n}\right\}$ in $T$ sucb that $\lim _{n \rightarrow \infty} x_{0} t_{n}=p$.

Proof. Sufficiency. The point $x_{0}$ in (i) is not almost periodic. If $x_{0}$ were almost periodic, then $\mathrm{Cl}\left(x_{0} T\right)$ is a minimal set [8, Theorem 4.07]. Since a minimal set is either a subset of $E(X)$ or a subset of $N(X)$ (use Theorem 1.2), we have a contradiction.

Necessity. By hypothesis there exists $x_{1} \in E(X)$ which is not almost periodic. In order to show (i), with $x_{1}$ for $x_{0}$, it suffices to show that $\mathrm{Cl}\left(x_{1} T\right) \not \subset$ $E(X)$. One verifies readily that an orbit closure which lies entirely in $E(X)$ must be a minimal set. Since $X$ is now assumed to be compact, such a minimal set is compact. In a compact minimal set every point is almost periodic [8, Theorem 4.05]. It follows from these reasonings that $\mathrm{Cl}\left(x_{1} T\right) \not \subset E(X)$.

The proof of Proposition 2.19 is completed.

2.20. Proposition. Let $(X, T)$ be a transformation group, where $X$ is compact connected. Suppose $N(X)$ is nonempty and semiclosed. Let

$$
\begin{aligned}
& \alpha=\max \left\{f_{T}(x) \mid x \in X\right\} \quad\left(\text { for } f_{T}\right. \text { see Remark 1.19), } \\
& \beta=\inf \left\{f_{T}(x) \mid x \in X\right\}, \\
& \gamma=\max \{\operatorname{dia}(C) \mid C \text { : components of } N(X)\} .
\end{aligned}
$$

The following properties bold:

(1) If $\gamma<\alpha$, then $E(X)$ bas a point which is not almost periodic.

(2) If $\gamma<\beta$, then for every component $C$ of $N(X)$ there exists $p \in C$ sucb that property (i) of Proposition 2.19 bolds.

Proof. (1) By hypothesis there exists $p_{0} \in N(X)$ such that $\gamma<f_{T}\left(p_{0}\right)$. Let $s$ be a real number such that $\gamma<s<f_{T}\left(p_{0}\right)$. The definition of the spread function is such that

$$
f_{T}(x)=\inf \left\{\sup \left\{\operatorname{dia}\left(S_{\epsilon}(x) t\right) \mid t \in T\right\} \mid \epsilon>0\right\}
$$

where $S_{\epsilon}(x)$ is the $\epsilon$-open ball around $x$. Hence there exist sequences $\left\{x_{n}\right\}$ and $\left\{y_{n}\right\}$ in $X$ and a sequence $\left\{t_{n}\right\}$ in $T$ such that

$$
\lim _{n \rightarrow \infty} x_{n}=p_{0}=\lim _{n \rightarrow \infty} y_{n}, \quad d\left(x_{n} t_{n}, y_{n} t_{n}\right)>s_{1}
$$

where $d$ is the metric for $X$. We may assume the existence of

$$
a=\lim _{n \rightarrow \infty} x_{n} t_{n}, \quad b=\lim _{n \rightarrow \infty} y_{n} t_{n} \text { and } q=\lim _{n \rightarrow \infty} p_{0} t_{n} \in N(X) .
$$

Then $d(a, b) \geq s$.

Now let $\left(X_{*}(S), T\right)$ be the $S$-partition transformation group of Definition 2.6 
with $S=N(X)$. Let $\pi: X \rightarrow X_{*}(S)$ be the $S$-projection. By the property that $\pi(x t)=\pi(x) t$ for all $x \in X, t \in T$, we have

$$
\begin{aligned}
\lim _{n \rightarrow \infty} \pi\left(x_{n}\right) & =\pi\left(p_{0}\right)=\lim _{n \rightarrow \infty} \pi\left(y_{n}\right), \\
\lim _{n \rightarrow \infty} \pi\left(x_{n}\right) t_{n} & =\pi(a) \neq \pi(b)=\lim _{n \rightarrow \infty} \pi\left(y_{n}\right) t_{n} .
\end{aligned}
$$

The inequality is due to the fact that $\gamma<s \leq d(a, b)$; hence no element in $X_{*}(s)$ can contain both $a$ and $b$. It follows from (a) that $\pi\left(p_{0}\right) \in N\left(X_{*}(S)\right)$. Now $N\left(X_{*}(S)\right)$ is totally disconnected. This is because of $N\left(X_{*}(S)\right) \subset \pi(S)$ by Proposition 2.7(4). By Proposition 1.3 the set $N\left(X_{*}(S)\right)$ is zero-dimensional. By Lemma 1.11 there exist some $y \in E\left(X_{*}(S)\right)$ and a sequence $\left\{t_{n}\right\}$ in $T$ such that $\lim _{n \rightarrow \infty} y t_{n}=\pi\left(p_{0}\right)$ : Let $x=$ $\pi^{-1}(y)$. Then $x \in E(X)$ and we must have $\lim _{n \rightarrow \infty} x t_{n} \in \pi^{-1}\left(\pi\left(p_{0}\right)\right)$. Property (i) of Proposition 2.19 is satisfied. The proof of (1) is now completed by applying Proposition 2.19.

The proof of (2) is similar. Here the point $p_{0}$ can be chosen from any component of $N(X)$.

The proof of Proposition 2.20 is completed.

Note that we use max instead of sup to define the numbers $a$ and $\gamma$ in Proposition 2.20. This is because the functions $f_{T}$ and dia are upper semicontinuous functions on compact spaces; hence they assume absolute maxima.

To illustrate the meaning of Proposition 2.20 we let $N(X)$ in (1) be totally disconnected. Then $\gamma$ in Proposition 2.20 is such that $\gamma=0$. The number $\alpha$ in Proposition 2.20 has the property that $\alpha=0$ if and only if $N(X)=\varnothing$. It follows from Proposition 2.20(1) that if $N(X)$ is totally disconnected and nonempty and if $X$ is compact connected, then $E(X)$ always has a point which is not almost periodic. This fact has been proved in Lemma 1.11, provided we take Proposition 2.19 into account. The application of Proposition 2.20 is, of course, intended for cases where $N(X)$ is not zero-dimensional.

3. Manifolds which carry SAE. In this section we apply $\$ \$ 1$ and 2 to obtain a result of transformation groups whose phase spaces are manifolds. It is shown in Theorem 3.4 that the only manifolds which can admit SAE (Definition 1.6) are topologically the standard ones, namely, $n$-cells, $n$-spheres, $n$-disks and closed upper half-planes of the euclidean $n$-spaces. The proofs make use of results of the generalized Schoenflies theorem. We remark that before us Kinoshita [17] has shown that if a closed $n$-manifold admits SAE for discrete flows then it is topologically an $n$-sphere.

We begin by simplifying the definition of SAE.

3.1. Proposition. Let $(X, T)$ be a transformation group, where $X$ is a connected metrizable n-manifold $(n \geq 2)$. Then $(X, T)$ is SAE if and only if the following conditions are satisfied: 
(a) $N(X)$ is nonempty and totally disconnected.

(b) For each $p \in N(X)$ the set $\mathrm{Cl}\left(p^{\prime} T\right)$ is compact.

Proof. It is shown in [20, Theorem 5.3] that if $X$ is a metrizable $n$-manifold $(n \geq 2)$ and if condition (a) holds, then $E(X)$ is indivisible by $T$. Hence (c) of Definition 1.6 can be omitted for this case.

3.2. Corollary. Let $(X, T)$ be a transformation group, where $X$ is a compact connected metrizable $n$-manifold $(n \geq 2)$. If $N(X)$ is totally disconnected, then it is compact and in fact it is either empty, a union of one or two points or a Cantor set.

Proof. By Proposition 3.1, $(X, T)$ with the given hypothesis is SAE. Corollary 3.2 then follows from some of the conclusions of Theorem 1.7.

Corollary 3.2 illustrates an advantage of our treatment of transformation groups with zero-dimensional $N(X)$ from the unified point of view of indivisibility, rather than from a particular topological point of view such as that $N(X)$ is closed and $E(X)$ is connected. If we follow the latter assumption, then the set $N(X)$ is assumed to be compact to begin with and the conclusion of Corollary 3.2 would not appear as strong as it is here.

3.3. Lemma. Let $(X, T)$ be SAE. For every $p \in N(X)$ there exists a sequence $\left\{t_{i}\right\}$ in $T$ with the following properties, depending on whether $X$ is compact:

(1) If $X$ is noncompact, then for each neighborbood $V$ of $p$ and compact set $K \subset X$ there exists a positive integer $m$ such that $K t_{i} \subset V$ for all $i \geq m$.

(2) If $X$ is compact, there exists some $q \in N(X)$ such that for each neighborbood $V$ of $p$ and compact set $K \subset X, q \notin K$, there exists a positive integer $m$ such that $\mathrm{Kt}_{i} \subset V$ for all $i \geq m$.

Proof. Statement (1) follows immediately from statement (2). If $X$ is noncompact we consider the on e-point compactification transformation group $\left(X^{*}, T\right)$ and apply (2) to $\left(X^{*}, T\right)$. From the statement of (2) and that $\infty$ is a fixed point disjoint from the fixed point $N(X)$, it is necessary that $q=\infty$. Hence $K$ becomes an arbitrary compact subset of $X$. Thus (2) implies (1).

(2) We apply Theorem 1.22 . We let $a$ in (1.22) be the point $p$ and let the sequence $\left\{s_{i}^{-1}\right\}$ of (1.22) be denoted by $\left\{t_{i}\right\}$. The point $b$ in (1.22) is our point $q$ in Lemma 3.3(2). Thus by Theorem 1.22 we have that

$$
\lim _{i \rightarrow \infty} x t_{i}=p \text { for all } x \neq q,
$$

and that $\left\{t_{i}\right\}$ is equicontinuous everywhere except $q$. Now let $V$ be a neighborhood of $p$ and $K$ a compact subset of $X, q \notin K$. For each $x \in K$ there exists a neighborhood $U_{x}$ of $x$ and a positive integer $i_{x}$ such that $U_{x} t_{i} \subset V$ for all 
$i \geq i_{x}$, due to the equicontinuity of $\left\{t_{i}\right\}$ at $x$ and equation (a). Since $K$ is compact, it is covered by a finite family $\left\{U_{x_{1}}, \cdots, U_{x_{n}}\right\}$ of such sets $U_{x^{*}}$. If we set $m=\max \left\{i_{x_{j}} \mid j=1,2, \cdots n\right\}$, then $K t_{i} \subset V$ for all $i \geq m$.

The proof of Lemma 3.3 is completed.

3.4. Theorem. Let $X$ be a connected metrizable n-manifold $(n \geq 2)$. If $X$ admits an SAE, i.e. with respect to a certain acting group $T$ and a certain metric of $X$ the set $N(X)$ is nonempty, totally disconnected and the orbit closure of its every point is compact, then the following properties bold.

(1) If $X$ is a closed manifold, then $X$ is topologically the n-sphere.

(2) If $X$ is an open manifold, then $X$ is an open $n$-cell.

(3) If $X$ is compact with boundary, then $X$ is a closed n-cell.

(4) If $X$ is noncompact with boundary, then $X$ is topologically the closed upper balf of the euclidean $n$-space.

Proof. (1) For proving this case the technique is identically the same as that used by Kinoshita [17] for proving discrete flows. Let $p,\left\{t_{i}\right\}$ and $q$ be defined as in Lemma 3.3(2). Let $V$ and $W$ be euclidean neighborhoods of $p$ and $q$ respectively. Let $K$ be the compact set $X-W$ and $m$ be the integer given in Lemma 3.3(2). By Lemma 3.3(2) we have $K t_{m} \subset V$. Hence $X=W \cup V t_{m}^{-1}$. Now any compact manifold which is the union of two open n-cells is lopologically the $n$-sphere (cf. [1] and [17]). This proves that $X$ is topologically an $n$-sphere.

(2) Let $p$ and $\left\{t_{i}\right\}$ be given as in Lemma 3.3(1). Let $V$ be an euclidean neighborhood of $p$ and $\mathrm{Cl}(V)$ be compact. Let $W$ be an open neighborhood of $\infty$ in $X^{*}$ such that $W \cap \mathrm{Cl}(V)=\varnothing$. Let $K$ be the compact set $X^{*}-W$ and $m$ the integer of Lemma 3.3(1) with respect to $V$ and $K$. Then $K t_{m} \subset V$. Hence $K \subset$ $V t_{m}^{-1} \subset \mathrm{Cl}(V) t_{m}^{-1}$. Let $V_{1}=V t_{m}^{-1}$. Then $V_{1}$ is an open $n$-cell and $V \subset K \subset V_{1}$. We repeat the previous argument with $V_{1}$ in place of $V$ and replace $W$ by a smaller neighborhood of $\infty$. We continue this process indefinitely and we let $W$ decrease to $\infty$. We then obtain a sequence of open $n$-cells

$$
V=V_{0} \subset V_{1} \subset V_{2} \subset \cdots, \text { with } X=\bigcup_{n=0}^{\infty} V_{n} \text {. }
$$

Now a union of monotonically increasing sequence of open n-cells is an open $n$-cell (cf. Brown [2]). Hence $X$ is an open $n$-cell.

(3) and (4). For these two cases we first show $N(X) \subset \partial X$, where $\partial X$ is the boundary of the manifold $X$. The set $\partial X$ is invariant under any transformation group built on $X$. Suppose now $(X, T)$ is SAE as assumed. Let $p$ be an arbitrary point in $N(X)$. It follows from Lemma 1.15 and the invariance of $\partial X$ that $p \in N(X)$. Hence $N(X) \subset \partial X$.

(3) We claim that $\partial X$ is homeomorphic to the $(n-1)$-sphere $S^{n-1}$. First 
$\partial X$ has a finite number of components, since $X$ is compact. Define a syndetic subgroup (also called uniform subgroup) $G$ of $T$ by $G=\{t \in T \mid C t=t$ for all components $C$ of $\partial X\}$. It is easy to verify that $N(X, T)=N(X, S)$. Also it is clear that $(X, G)$ is SAE. Let $p \in N(X)$ and $C_{p}$ be the component of $\partial X$ which contains $p$. We now apply Lemma 1.15 with $G$ in place of $T$. Since a component of $\partial X$ has more than one point and components of $\partial X$ are invariant under $G$, Lemma 1.15 shows that $C_{p}$ is the only component of $\partial X$. Hence $\partial X$ is connected and is therefore a connected closed $(n-1)$-manifold. If $n=2$, then $\partial X$ is a compact connected metrizable 1 -manifold without boundary and is then necessarily a topological circle. If $n \geq 3$ then $n-1 \geq 2$ and Theorem 3.4(1) implies that $\partial X$ is topologically $S^{n-1}$. For all cases we have that $\partial X$ is topologically $S^{n-1}$.

We now make the doubling manifold $X^{2}$ of $X$, which is obtained by making two disjoint copies of $X$ and identifying points in the boundaries in the canonical way. Give an arbitrary metric for the compact metrizable space $X^{2}$. The transformation group $(X, T)$ extends canonically to a transformation group $\left(X^{2}, T\right)$. It is readily verified that $\left(X^{2}, T\right)$ is SAE. In fact, $N\left(X^{2}\right)=N(X)$. Since $X^{2}$ is a closed $n$-manifold we may apply Theorem $3.4(1)$ to conclude that $X^{2}$ is topologically $S^{n}$. The space $X$ is then a topological $n$-disk. Thus we may regard $X^{2}$ as a standard $n$-sphere $S^{n}$. Then $\partial X$ is the image of a locally flat embedding of $S^{n-1}$ into $S^{n}$. This embedding can be extended to $S^{n-1} \times$ $(-1,1) \rightarrow S^{n}$ (embedding) (cf. [3, Theorem 3]). From here on the assertion that $X$ is a closed $n$-cell is the direct consequence of the generalized Schoenflies theorem proved independently by M. Morse [22] and M. Brown [1] (also see their references on B. Mazur).

(4) By Theorem 1.28 we know that $N(X)$ is a fixed point $p$. Hence the component $C_{p}$ of $\partial X$ which contains $p$ is invariant and all other components consist of equicontinuous points. It follows from Lemma 1.15 and the fact that elements of $T$ take components of $\partial X$ to components of $\partial X$ that $C_{p}$ is the only component of $\partial X$. If we extend $(X, T)$ to $\left(X^{*}, T\right)$ then $\left(X^{*}, T\right)$ is SAE (Theorem 1.27) and $N\left(X^{*}\right)=\{\infty\} \cup N(X)=\{\infty, p\}$. If we apply Corollary 1.29 to $\left(X^{*}, T\right)$ we see that $X$ contains no nonempty compact invariant subset other than $\{p\}$. In particular, the set $\partial X$ is noncompact. The set $\partial X$ is then an open $(n-1)$-manifold. If $n>2$ we apply Theorem $3.4(2)$ to $(\partial X, T)$ to conclude that $\partial X$ is an open $(n-1)$-cell. If $n=2$, then $\partial X$ is a noncompact connected metrizable 1 -manifold without boundary and is necessarily a topological open interval, which is then also an open $(n-1)$-cell.

We form the doubling manifold $X^{2}$ as in the proof of (3) and extend $(X, T)$ to $\left(X^{2}, T\right)$ canonically. Give $X^{2}$ an induced metric from its one-point compactification $\left(X^{2}\right)^{*}$. A proof similar to that of Proposition 1.1(5) shows that the 
nonequicontinuous set $N(X)$ is unchanged when the original metric is replaced by the induced metric from $\left(X^{2}\right)^{*}$. Likewise if $Y$ is the other copy of $X$ which together with $X$ forms $X^{2}$, then $N(Y)$ is unchanged when the original metric is replaced by the induced metric from $\left(X^{2}\right)^{*}$. It follows that $N\left(X^{2}\right)=\{p\}$. In particular $\left(X^{2}, T\right)$ is SAE. Since $X^{2}$ is an open $n$-manifold which carries SAE, it is necessarily an open $n$-cell by Theorem 3.4(2).

We now show that $X$ is topologically the closed half-plane of $\mathbf{R}^{2}$. We observe that $\left(X^{2}\right)^{*}$ is topologically the $n$-sphere $S^{n}$. Let $\left(X^{2}\right)^{*}=\{\infty\} \cup X^{2}$. Then $\{\infty\} \cup \partial X$ is a topological $(n-1)$-sphere which we denote as $\Sigma^{n-1}$. There is no loss of generality in assuming that $\left(X^{2}\right)^{*}$ is the standard $n$-sphere $S^{n}$. Hence there exists an embedding $f: S^{n-1} \rightarrow S^{n}$ such that $f\left(S^{n-1}\right)=\Sigma^{n-1}$. This embedding is locally flat everywhere except possibly at $\infty \in S^{n}$. We separate cases according to the dimension $n$ of $X$. If $n=2$ the previous discussion clearly shows that $X$ is topologically the closed half of $\mathbf{R}^{2}$. For $n=3$ we use the following theorem of Harrold-Moise [10]: Let $K$ be a topological 2-sphere in $S^{3}$ that is locally flat except at a single point $p$ and let $A$ and $B$ be the complementary domains; then either $\mathrm{Cl}(A)$ or $\mathrm{Cl}(B)$ is a closed 3-cell. In our case $K=\Sigma^{2}$. Hence either $X \cup\{\infty\}$ or $Y \cup\{\infty\}$ is a closed 3-cell. Hence either $X$ or $Y$ is topologically the closed half of $\mathbf{R}^{3}$. Since $Y$ is just a copy of $X$, this proves the case $n=3$. For $n>3$ we use the following theorem of Cantrell [4]: Let $S$ be a topological $(n-1)$-sphere in $S^{n}(n>3), p \in S$ and $G$ a component of $S^{n}-S$. Suppose that $S$ bas a local collar in $\mathrm{Cl}(G)$ at each point of $S-\{p\}$; then $\mathrm{Cl}(G)$ is a closed $n$-cell. Hence $\{\infty\} \cup X$ is a closed $n$-cell for $n>3$. It follows that $X$ is topologically the closed half-plane of $\mathbf{R}^{n}$ for $n>3$. This completes all cases for (4).

The proof of Theorem 3.4 is completed.

3.5. Remark. (1) Theorem 3.4 is valid also for the case $n=1$ for SAE. The equivalent property for SAE stated in (3.4) should now include that $E(X)$ is indivisible by $T$. The same proof for (3.4) applies for this special case.

(2) The converse of Theorem 3.4 also bolds, i.e., the four types of manifolds given in (3.4) admit SAE. It is easy to see that each such manifold carries discrete flows and continuous flows which are SAE. Sucb transformation groups with other acting groups can also be constructed.

(3) Theorem 3.4 has been announced as Theorem 9 in [19]. Part (IV) of [19, Theorem 9] is incorrect and is now corrected as (4) of Theorem 3.4.

Added in proof. Since this paper was written, some literature on the subject of almost equicontinuous transformation groups bas appeared in publication. Particularly we mention that F. B. Roberson obtained independently some results related to Theorem 1.28 bere or Theorem 5 of our announcement [19]. 
Roberson arrived at the conclusion that $N(X)$ is a fixed point for uniform spaces, but with a bypothesis which is more restricted than that of Theorem 1.28 when the phase spaces are metric spaces. The results of Roberson appeared in "A theorem on the near equicontinuity of transformation groups", Proc. Amer. Matb. Soc. 27 (1971), pp. 189-191 and in "Some theorems on the structure of nearly equicontinuous transformation groups", Canad. J. Matb. 23 (1971), pp. 421-425. We also mention that a number of other results on almost regular bomeomorphisms on manifolds have been obtained by L. S. Husch since the works of [5], [13].

We thank Professors W. Gottschalk, S. Kinoshita, M. Morse and R. Summerhill for discussions.

\section{REFERENCES}

1. Morton Brown, A proof of the generalized Schoenflies theorem, Bull. Amer. Math. Soc. 66 (1960), 74-76. MR $22 \# 8470$ b.

2. - The monotone union of open n-cells is an open n-cell, Proc. Amer. Math. Soc. 12 (1961), 812-814. MR 23 \#A4129.

3. - Locally flat imbeddings of topological manifolds, Ann. of Math. (2) 75 (1962), 331-341. MR 24 \#A3637.

4. J. C. Cantrell, Non-flat embeddings of $S^{n-1}$ in $S^{n}$, Michigan Math. J. 10 (1963), 359-362. MR $27 \# 6274$.

5. P. F. Duvall, Jr. and L. S. Husch, Taming irregular sets of homeomorphisms, Bull. Amer. Math. Soc. 78 (1972), 77-79.

6. S. Eilenberg, Sur les transformations périodiques de la surface de sphère, Fund. Math. 22 (1934), 28-41.

7. W. H. Gottschalk, Substitution minimal sets, Trans. Amer. Math. Soc. 109 (1963), 467-491. MR $32 \# 8325$.

8. W. H. Gottschalk and G. A. Hedlund, Topological dynamics, Amer. Math. Soc. Colloq. Publ., vol. 36, Amer. Math. Soc., Providence, R. I., 1955. MR 17, 650.

9. W. J. Gray and F. A. Roberson, On the near equicontinuity of transformation groups, Proc. Amer. Math. Soc. 23 (1969), 59-63. MR 39 \#7583.

10. O. G. Harrold, Jr. and E. E. Moise, Almost locally polyhedral spheres, Ann. of Math. (2) 57 (1953), 575-578. MR 14, 784.

11. T. Homma and S. Kinoshita, On homeomorphisms which are regular except for a finite number of points, Osaka Math. J. 7 (1955), 29-38. MR 16, 1140.

12. W. Hurewicz and H. Wallman, Dimension theory, Princeton Math. Series, vol. 4, Princeton Univ. Press, Princeton, N. J., 1941. MR 3, 312.

13. L. S. Husch, $A$ topological characterization of the dilation in $E^{n}$, Proc. Amer. Math. Soc. 28 (1971), 234-236. MR $44 \# 1051$.

14. L. S. Husch and P.-F. Lam, Homeomorphisms of manifolds with zero-dimensional sets of recurrent points (to appear).

15. S. K. Kaul, On almost regular homeomorphisms, Canad. J. Math. 20 (1968), 1-6. MR $36 \# 5908$.

16. B. V. Kerékjártó, Topologische Charakterisierung der linearen Abbildungen, Acta. Sci. Math. (Szeged) 6 (1934), 235-262. 
17. S. Kinoshita, On quasi-translations in 3-sphere, Fund. Math. 56 (1964), 69-79. MR $30 \# 1502$.

18. K. Kuratowski, Topology. Vols. 1, 2, Academic Press, New York, 1966, 1968. MR $36 \# 8405 ; 41$ \#4467.

19. Ping-Fun Lam, On a theorem of B. von Kerékjártó, Bull. Amer. Math. Soc. 77 (1971), 230-234. MR $42 \# 2459$.

20. - Equicontinuity and indivisibility in transformation groups, Trans. Amer. Math. Soc. 174 (1972), 399-424.

21. D. Montgomery and L. Zippin, Topological transformation groups, Interscience, New York, 1955. MR 17, 383.

22. M. Morse, A reduction of the Schoenflies extension problem, Bull. Amer. Math. Soc. 66 (1960), 113-115. MR 22 \#8470a.

23. A. Pfluger, Theorie der Riemannschen Flächen, Springer-Verlag, Berlin, 1957. MR 18, 796.

24. S. Smale, Differential dynamical systems, Bull. Amer. Math. Soc. 73 (1967), 747-817. MR 37 \#3598.

25. G. T. Whyburn, Analytic topology, Amer. Math. Soc. Colloq. Publ., vol. 28, Amer. Math. Soc., Providence, R. I., 1942. MR 4, 86.

SCHOOL OF MATHEMATICS, INSTITUTE FOR ADVANCED STUDY, PRINCETON, NEW JERSEY 08540

DEPARTMENT OF MATHEMATICS, UNIVERSITY OF MISSOURI, COLUMBIA, MISSOURI 65201 (Current address) 